\title{
Des premières amours aux secondes
}

Ernst Haeckel, de ses débuts en botanique à sa conversion à la zoologie

\section{Jens Pahnke}

Traducteur : Elvyre Gobert et Nicolas Wanlin

\section{(2) OpenEdition}

\section{Journals}

Édition électronique

URL : http://journals.openedition.org/aes/1116

DOI : 10.4000/aes. 1116

ISSN : 2258-093X

\section{Éditeur}

Laboratoire LISAA

\section{Référence électronique}

Jens Pahnke, «Des premières amours aux secondes », Arts et Savoirs [En ligne], 9 | 2018, mis en ligne le 14 mai 2018, consulté le 23 avril 2019. URL : http://journals.openedition.org/aes/1116 ; DOI :

10.4000/aes. 1116

Ce document a été généré automatiquement le 23 avril 2019

Centre de recherche LISAA (Littératures SAvoirs et Arts) 


\section{Des premières amours aux secondes}

Ernst Haeckel, de ses débuts en botanique à sa conversion à la zoologie

Jens Pahnke

Traduction : Elvyre Gobert et Nicolas Wanlin

Introduction 
Ernst Haeckel (1834-1919) ne fut pas seulement un zoologiste remarquable du $\mathrm{XIX}^{\mathrm{e}}$ siècle, renommé pour ses travaux précurseurs sur les radiolaires, les méduses et les éponges calcaires. Il fut également, par ses célèbres travaux, un ardent défenseur, aussi bien admiré que contesté, de la théorie darwinienne de l'évolution et $\mathrm{du}$ monisme. Avec sa Generelle Morphologie der Organismen (Morphologie générale des organismes), il retraça en 1866 l'histoire de la biologie et conçut à nouveaux frais les sciences de la vie sur des fondements

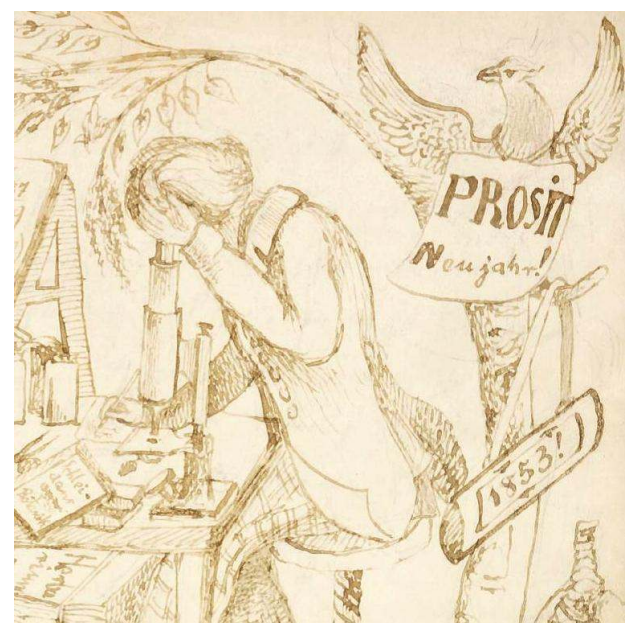
évolutionnistes. Nombre de notions couramment utilisées de nos jours nous viennent de Haeckel : l'ontogénie, la phylogénie, l'écologie - pour n'en citer que trois. De 1861 à 1909, il fut professeur à l'université de Iéna où il créa l'Institut de zoologie, qui connut par la suite une renommée mondiale. Le Phyletische Museum (musée de la phylogénie) qu'il fonda en 1908 ainsi que son ancienne demeure, la Villa Medusa, sont aujourd'hui des lieux dédiés à la recherche internationale. Mais Haeckel se fit aussi connaître en tant qu'artiste. Dans les Kunstformen der Natur (1899-1904) (Formes artistiques de la nature), il exposa de façon saisissante la diversité des organismes et influença ainsi de nombreux domaines, de l'art contemporain jusqu'à l'architecture. Haeckel mariait adroitement l'art et la science, notamment au fil de ses grands voyages dont il savait tirer de captivants récits. Sa personnalité était composée de ces nombreuses facettes ${ }^{1}$.

2 L'une de ces facettes, Haeckel botaniste, n'est que rarement évoquée par les commentateurs. Il a pourtant lui-même dit de la botanique qu'elle était son premier amour. La zoologie ne constituait ainsi que sa deuxième passion, mais elle sut le détourner de la scientia amabilis grâce au nouvel engouement que favorisa Johannes Müller ${ }^{2}$.

Enfant, Haeckel montrait déjà un intérêt prononcé pour les plantes. Tout au long de sa scolarité à Mersebourg de 1840 à 1852 sous l'égide de ses professeurs, au sein de son cercle d'amis, et enfin sous l'influence des œuvres de Matthias Jakob Schleiden et d'Alexander von Humboldt, une grande passion pour la botanique se développa chez Haeckel, au point qu'il voulut en faire son métier et poursuivre ses études avec Schleiden à Iéna. Pendant ses études de médecine à Wurtzbourg et à Berlin, de 1852 à 1854, son intérêt se tourna vers la zoologie. Pour quelles raisons le si enthousiaste " homme des plantes » („Pflanzenmenschen“) - ainsi que le surnommaient ses parents - n'est-il pas devenu le botaniste Ernst Haeckel ? Pourquoi s'orienta-t-il vers la zoologie, son deuxième amour? Et quelle place accorder, dans sa vie et son œuvre, à ses études botaniques? Jusqu'à présent, ces questions ont à peine été traitées. On peut toutefois trouver quelques éléments de réponse sous la propre plume de Haeckel, puisqu'il qualifiait ses débuts en botanique "d'études infantiles » et laisse ainsi l'impression qu'il s'agissait simplement des premiers pas d'un jeune garçon versé dans l'histoire naturelle. Les études botaniques de Haeckel eurent en réalité une portée bien plus importante que ce qui a été admis jusqu'à présent. 
Heinrich Schmidt ${ }^{3}$ et Georg Hecht ${ }^{4}$ sont les seuls à s'être intéressés de plus près au botaniste Haeckel. Tandis que Schmidt s'est appuyé en grande partie sur les dernières esquisses autobiographiques inédites de Haeckel (Lebenswege Ernst Haeckels ${ }^{5}$ ), Hecht a exploité pour son étude sur le voyage de 1852 à Teplice la correspondance, les journaux et les herbiers de Haeckel ${ }^{6}$. Grâce au projet "Ernst Haeckel (1843-1919): édition de la correspondance », les lettres envoyées par Haeckel et adressées à lui sont, depuis 2013, en cours de retranscription et de publication dans leur intégralité 7 . Elles permettent d'avoir un aperçu de ses travaux de collecte, de ses excursions, des lieux de découverte des plantes, de ses études botaniques à Berlin et à Wurtzbourg ainsi que de son intérêt progressif pour la zoologie. Ses carnets de voyage inédits et ses journaux permettent à Schmidt et Hecht d'affirmer que Haeckel était bien plus qu'un simple botaniste amateur. Un herbier aussi important - dont Schmidt estima le nombre de plantes à $12000^{8}$ - ne saurait être qualifié « d'études botaniques infantiles ».

5 Ce qui suit est un résumé du déroulement des études botaniques de Haeckel entre 1840 et 1854 qui expose et questionne les raisons de son intérêt pour la zoologie. Pour cela, on donnera la parole à Haeckel lui-même, autant que possible, sous forme d'extraits de sa correspondance et de ses journaux. Ce regard posé sur ses études botaniques offrira un aperçu d'une facette presque délaissée de sa biographie qui a pourtant influencé ses autres travaux.

\section{Les études botaniques « infantiles » à Mersebourg}

6 Ernst Haeckel naquit le 19 février 1834 à Potsdam, mais sa famille ${ }^{9}$ déménagea très vite, en 1835, à Mersebourg où son père Carl Gottlob Haeckel avait un emploi d'administrateur civil. Sa rencontre avec la botanique se produisit dès la petite enfance, à l'âge de six ans, et occupa chez lui une place déterminante jusqu'à ses vingt ans. Dans cette famille plutôt libérale, l'instruction était d'une importance majeure. Avant que Haeckel n'intègre l'école publique, sa mère Charlotte lui avait déjà appris à lire et à écrire. Sa rencontre avec Karl Gude $^{10}$ fut alors décisive :

Cet homme pur et prodigieux était un simple instituteur qui ne jouissait d'aucune formation universitaire mais qui avait tâché, autant que possible, de pallier ce manque en étant un habile autodidacte, notamment dans le domaine des sciences naturelles. À cet égard, je lui dois une immense reconnaissance, puisqu'il fut le premier à m'initier avec un esprit profond, bienveillant et singulier aux études auxquelles j'allais dédier ma vie : les sciences naturelles dans leur ensemble et tout particulièrement la botanique. ${ }^{11}$

Professeur particulier de Haeckel, dès ses six ans, il cultiva ses compétences en calcul. Mais très vite son enseignement alla bien au-delà des mathématiques. En effet, il encourageait systématiquement l'intérêt de Haeckel pour la nature et enseigna à son jeune élève, dès l'âge de huit ans, à identifier et à disséquer les plantes. Enfin ils réalisèrent ensemble, au fil de nombreuses excursions en pleine nature, son premier herbier ${ }^{12}$. 
Fig. 1
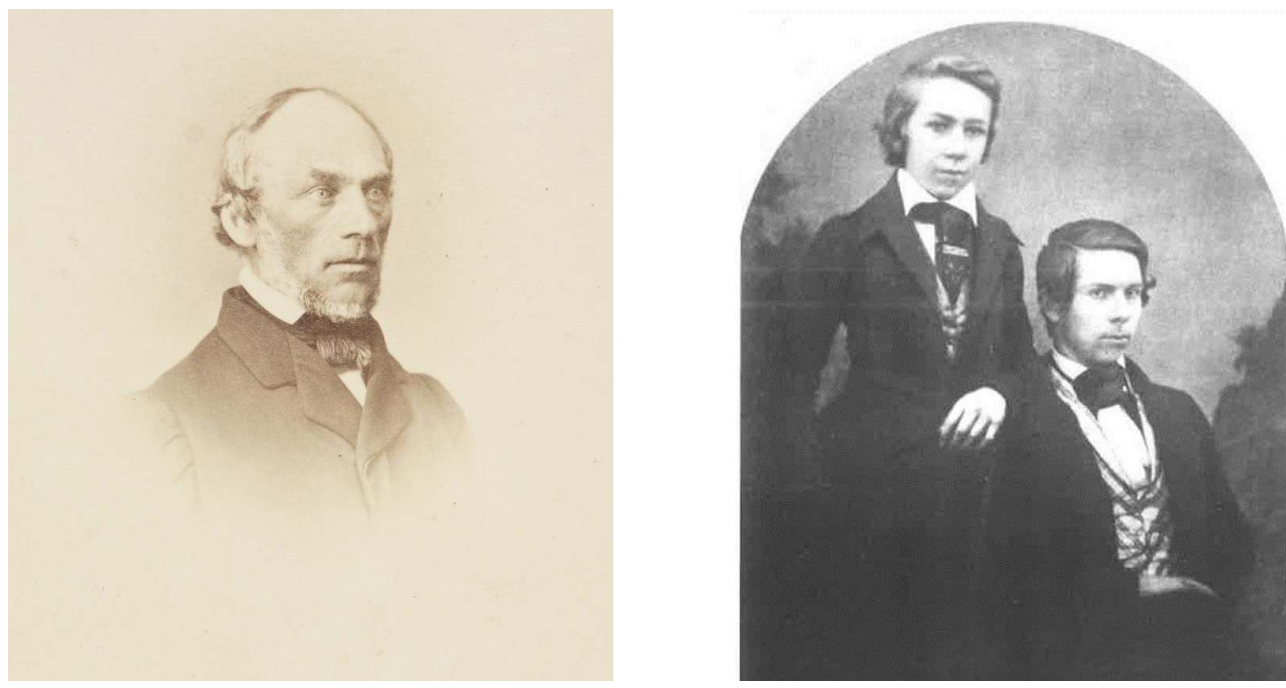

Figure de gauche : Karl Gude (1814-1898) photographié par le frère Ochs (à Magdebourg), en 1860 ; Figure de droite : Ernst Haeckel (à gauche) avec son frère Karl (à droite) À Mersebourg en 1849 Archives Ernst Haeckel à léna, désormais AEHI

Haeckel ne s'arrêta pas à ses petites "études botaniques infantiles » et continua à travailler sous la houlette de Gude sur la systématique des plantes.

La botanique prévalait désormais sur tous les autres centres d'intérêt de ma prime jeunesse : je procédais à la récolte et au séchage des plantes locales ainsi qu'à leur classement systématique dans un herbier riche et varié. Au début, j’utilisai le petit ouvrage de Kittel pour m'aider à la classification, plus tard je m'appuyai davantage sur le Cürie ainsi que sur la Flora Deutschlands de Koch. ${ }^{13}$

Leurs expéditions eurent une grande importance dans le cadre de ce programme d'étude. En 1845, ils firent tous les deux un voyage dans le Harz, où ils logèrent chez les parents de Gude, pour y faire de grandes excursions consacrées à l'histoire naturelle ${ }^{14}$. À côté de l'incontournable récolte de plantes, la géographie et l'histoire du pays étaient étudiées et Haeckel affuta sa manière d'observer les paysages. Une autre passion fut également très tôt encouragée : le dessin. Sous l'instruction de son professeur de dessin, Oscar Naumann ${ }^{15}$, Haeckel développa une aptitude surprenante à reproduire paysages et objets. L'écolier Haeckel avait déjà un intérêt certain, et qui avait été fortement attisé, pour l'histoire naturelle, les voyages et l'art.

\section{L'« homme des plantes » („Pflanzenmensch“)}

10 Après son entrée au Domgymnasium (lycée de la cathédrale) de Mersebourg au printemps 1843, Haeckel étudia les plantes avec encore plus d'ardeur. Il trouva d'ailleurs un nouveau soutien auprès d'un de ses professeurs: Otto Gandtner ${ }^{16}$. Gandtner cultiva le goût de Haeckel pour les plantes et entreprit de faire des randonnées botaniques avec lui et d'autres élèves qui s'y intéressaient également. Haeckel put partager sa passion pour la botanique jusqu'à la fin de sa scolarité avec trois camarades : Ernst Weiss, Victor Weber et Wilhelm Hetzer ${ }^{17}$. Ensemble, ils formaient le «trèfle botanique " (,Botanische Kleeblatt"), ils partaient tous les trois en excursion pour trouver des plantes rares et s'échangeaient certaines espèces. 
Fig. 2a

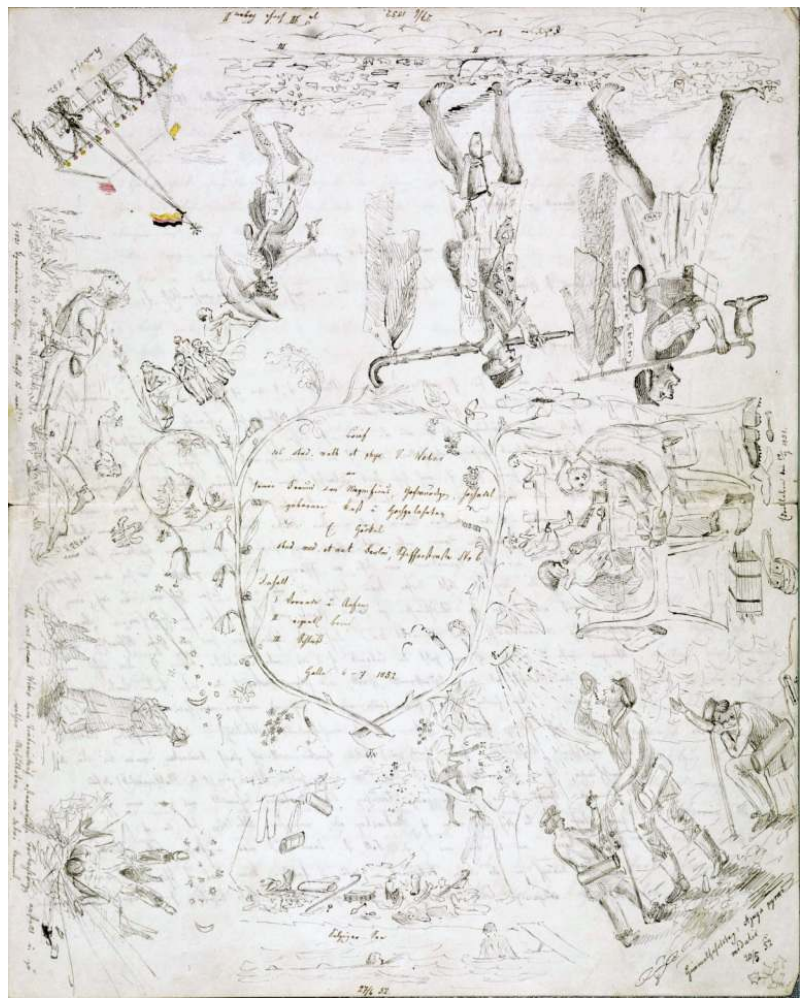

Victor Weber, lettre à Ernst Haeckel du 6 juillet 1852. Avec les dessins de Weber en souvenir de leurs excursions botaniques, AEHI, cote A 16209

$\mathrm{AEHI}$

Fig. 2b

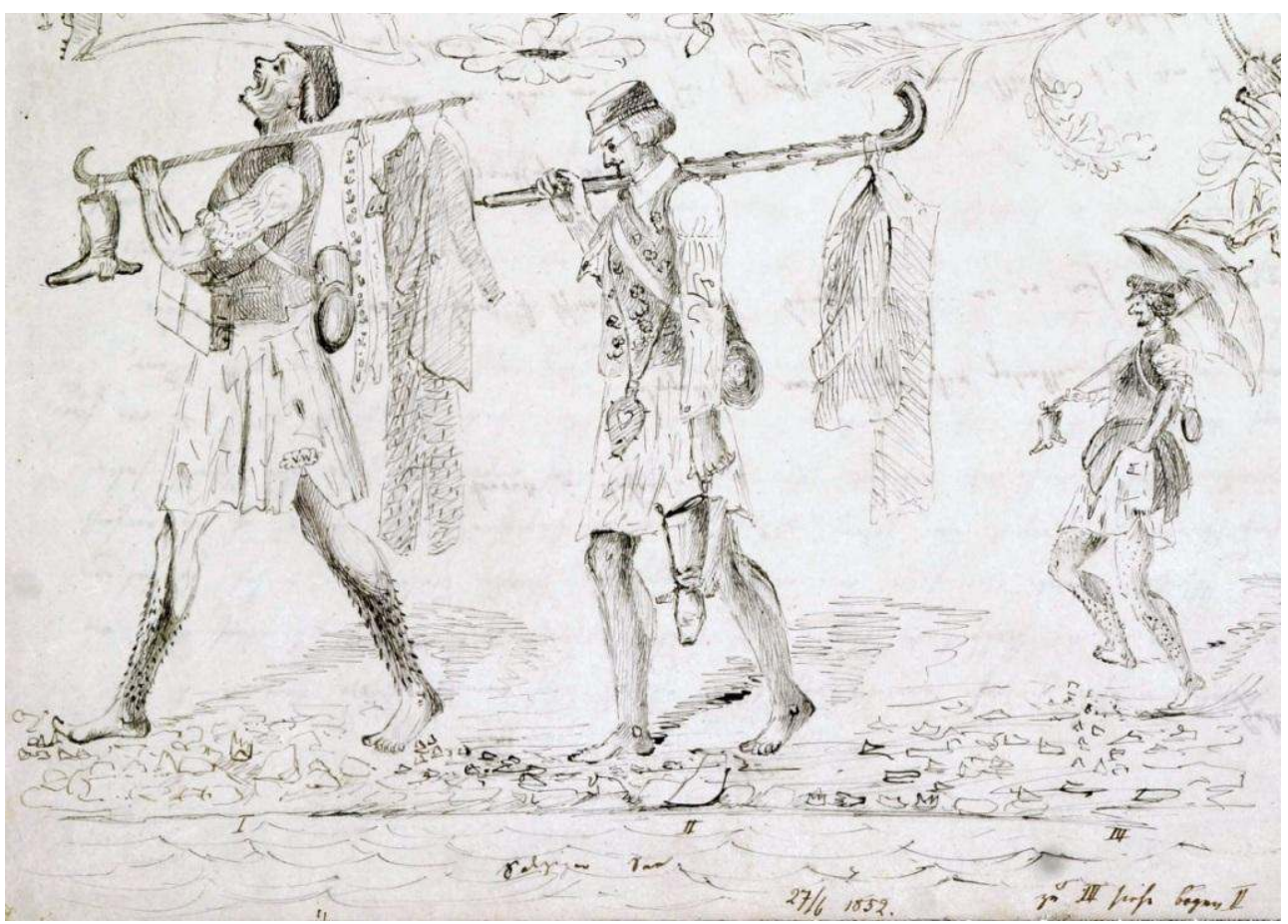

„Salziger See 27/6 1852", Weber (à gauche) avec Haeckel (à droite) en excursion 
Fig. 2c

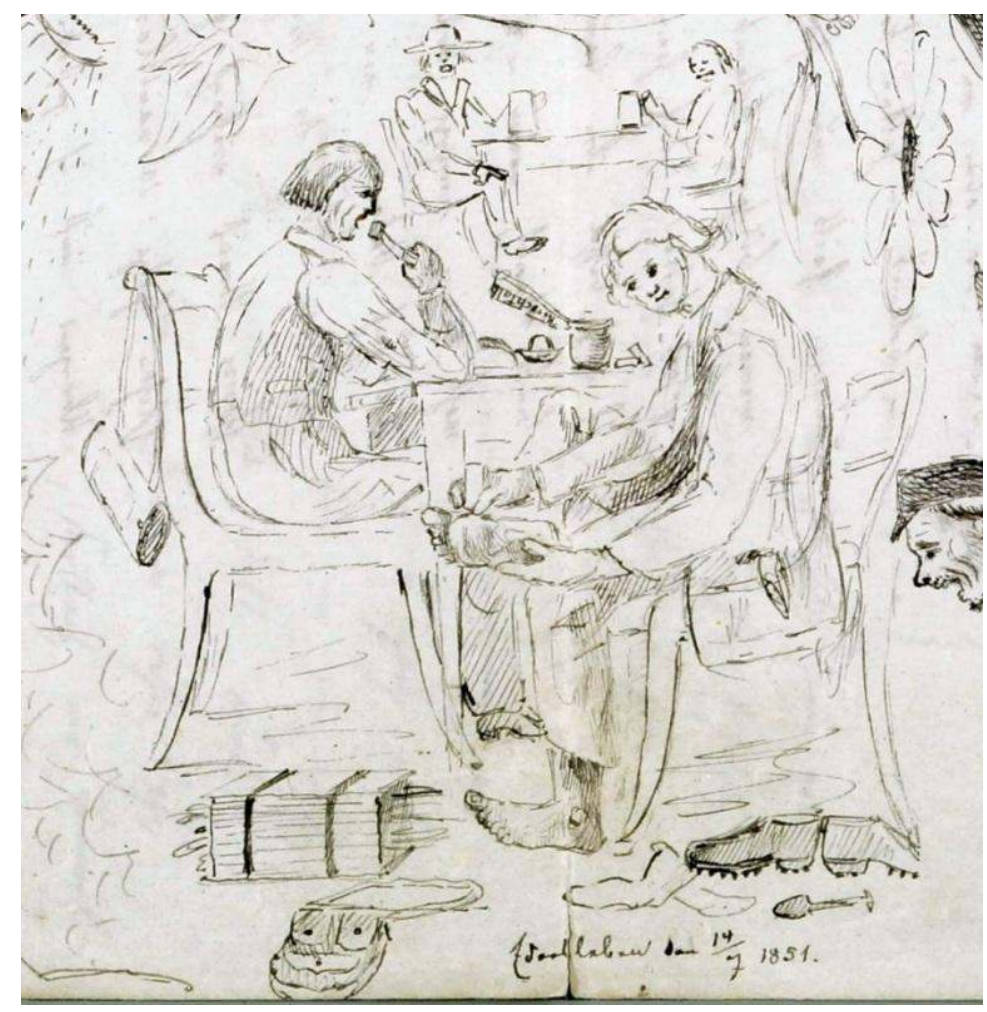

„Edersleben 14/7 1851“, Weber (à gauche) et Haeckel (à droite) lors d'une halte

11 Une lettre de Victor Weber ${ }^{18}$ à Ernst Haeckel, retranscrivant certains événements de l'été 1851 sous forme de dessins, donne un aperçu de ce à quoi pouvaient ressembler les excursions botaniques de Haeckel. On peut les voir tous deux équipés de boîtes et de presses de botanistes et de petites bêches pour déterrer les plantes. Les randonnées n'étaient pas de tout repos. On peut notamment voir Haeckel s'occuper de ses pieds couverts d'ampoules ouvertes lors d'une halte (Fig. 2c). Empruntant des voies impraticables, ils y laissèrent des lambeaux de vêtements! Les excursions n'étaient pas seulement dédiées à la récolte des plantes mais laissaient également place à l'aventure. Les botanistes en herbe pouvaient ainsi faire eux-mêmes l'expérience de ce qu'ils avaient pu lire dans les carnets de voyage de Darwin et de Humboldt. Haeckel réunit une centaine de plantes dans un herbier spécial, le Memorial-Herbarium ${ }^{19}$, qui demeura comme un souvenir de ce dernier été de ses années de lycée. Mais ses connaissances de la flore locale constituaient également pour lui un point d'entrée dans la littérature spécialisée. Il put ainsi transmettre à August Garcke la localisation de quelques espèces rares de la flore locale. Par la suite, Haeckel resta toujours en contact avec Garcke pour échanger sur les plantes de son herbier ${ }^{20}$. Ludwig Finsterbush ${ }^{21}$, un des plus proches amis d'enfance de Haeckel, a également souligné la valeur de cet herbier: «Un lycéen de Mersebourg n'avait certainement encore jamais constitué un tel herbier, exceptionnel aussi bien du point de vue qualitatif que quantitatif. ${ }^{22}$ Pour faire cela dans les règles de l'art, Haeckel constitua même un herbier annexe, puisqu'il lui était parfois difficile de classer les plantes singulières de la « bonne manière $»^{23}$. À ce sujet, Haeckel écrivit ceci :

Pendant toute ma scolarité, l'élaboration de mon herbier se trouva au tout premier plan de mes intérêts et absorba la plus grande partie de mon temps libre, si bien 
que je voudrais dire quelques mots sur les bénéfices de cette pratique, d'autant plus que plus tard j'ai souvent blâmé certains aspects de l'herbier botanique. ${ }^{24}$

La botanique occupait presque tout le temps libre dont disposait Haeckel après l'école. Après ses excursions, il rentrait souvent épuisé à la maison, mais il s'occupait alors de ses plantes pendant des heures et les observait au microscope. Ce n'est pas sans inquiétude que ses parents regardaient le zèle de leur fils se transformer en réel fanatisme. Il en résultait d'ailleurs régulièrement quelques tensions, particulièrement entre Haeckel et son père, parce que ce dernier ne voyait pas dans l'étude de la botanique les fondements vraiment solides pour une profession future qui lui permettrait de gagner sa vie convenablement. Néanmoins, consacrer autant de temps aux plantes avait aussi des côtés positifs. En effet, en travaillant sur son herbier, il développait des facultés particulières telles que l'observation minutieuse et la dissection des plantes, il apprenait une terminologie spécifique et la classification dans un système. C'est finalement à cet entraînement quotidien que Haeckel dut ses capacités d'observation si phénoménales et son sens du détail mis en œuvre dans ses travaux ultérieurs. Enfin, la « récolte de foin ", comme Haeckel la nommait, recélait en plus d'une grande valeur esthétique, une beauté que les plantes ne montrent pas, une valeur sentimentale. Ses plantes séchées constituent, au même titre que ses lettres et ses journaux, d'importants jalons de sa biographie :

Tant de beaux souvenirs, s'étalant sur un demi-siècle, sont liés à cette " récolte de foin ». [...] Cette cueillette de fleurs et cette collection d'herbes témoignent d'un attrait et d'un foisonnement d'idées des plus variées comme il ne peut peut-être en résulter d'aucun autre exercice. ${ }^{25}$

Fig. 3a

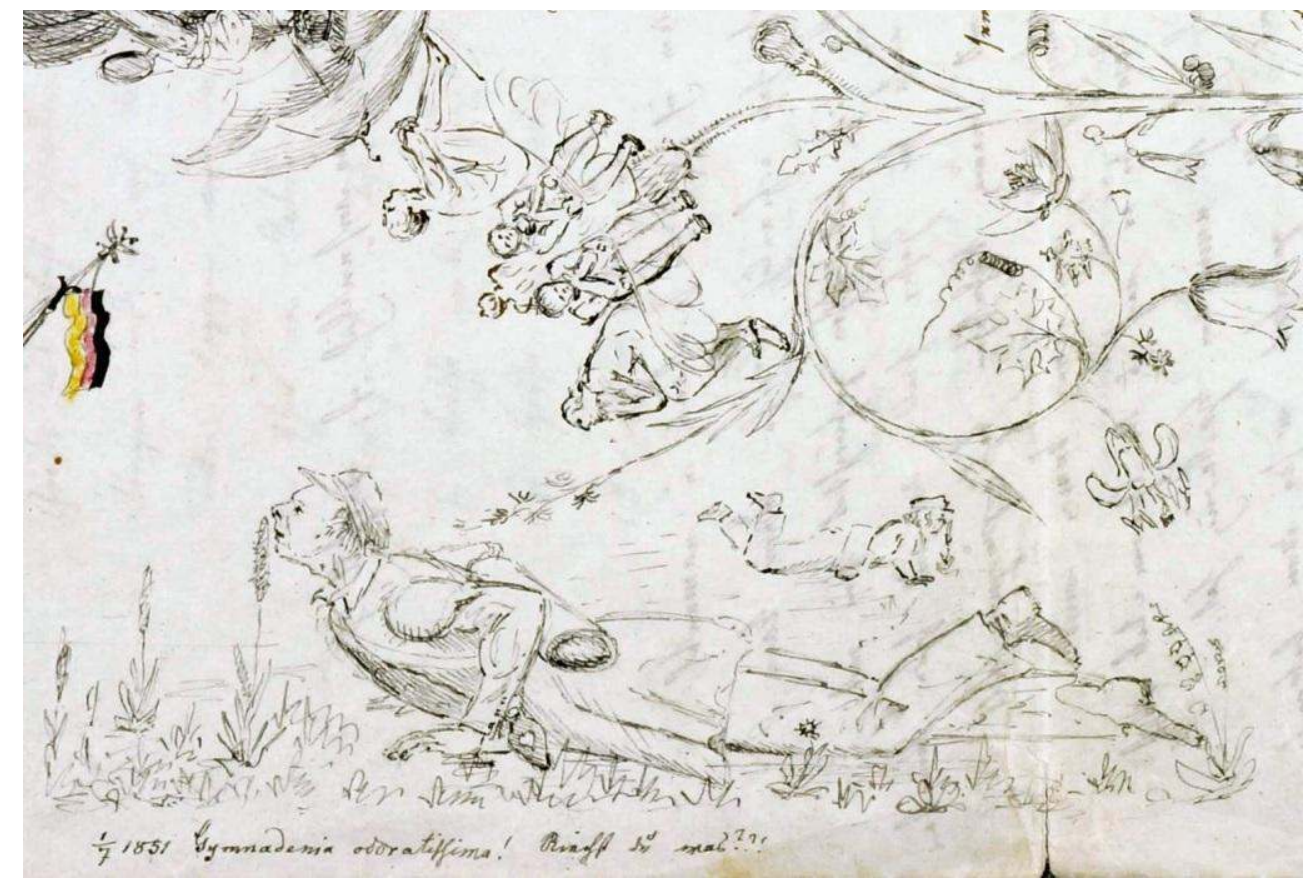

Victor Weber, lettre à Ernst Haeckel du 6 Juillet 1852, «1/7 1851 Gymnadenia odoratissima! Sens-tu quelque chose? ? ! », AEHI, cote A 16209

$\mathrm{AEHI}$ 
Fig. 3b

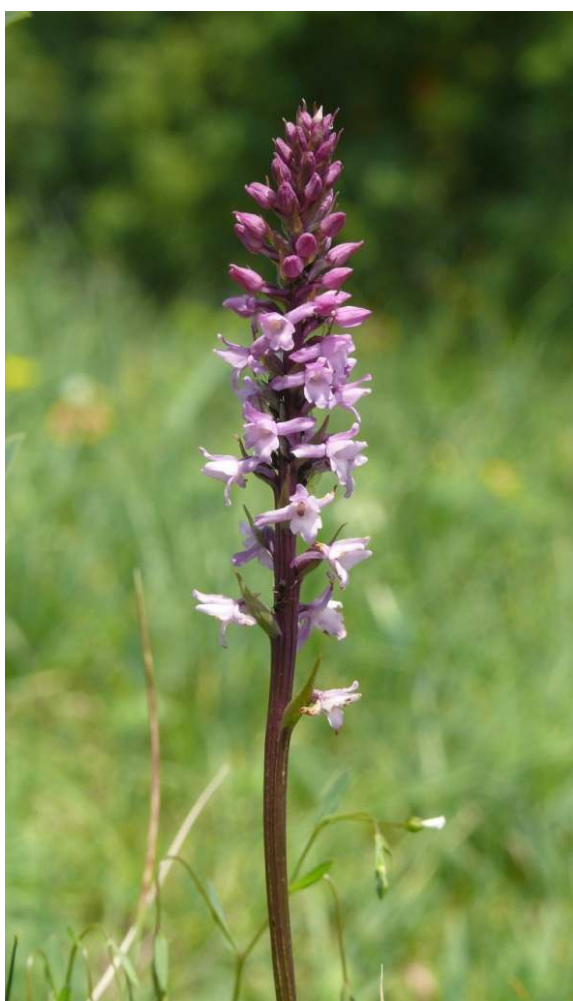

Orchis Odorant, Gymnadenia odoratissima L. (Rich)

(c) Wikimedia Commons, BerndH 2010 
Fig. 3c

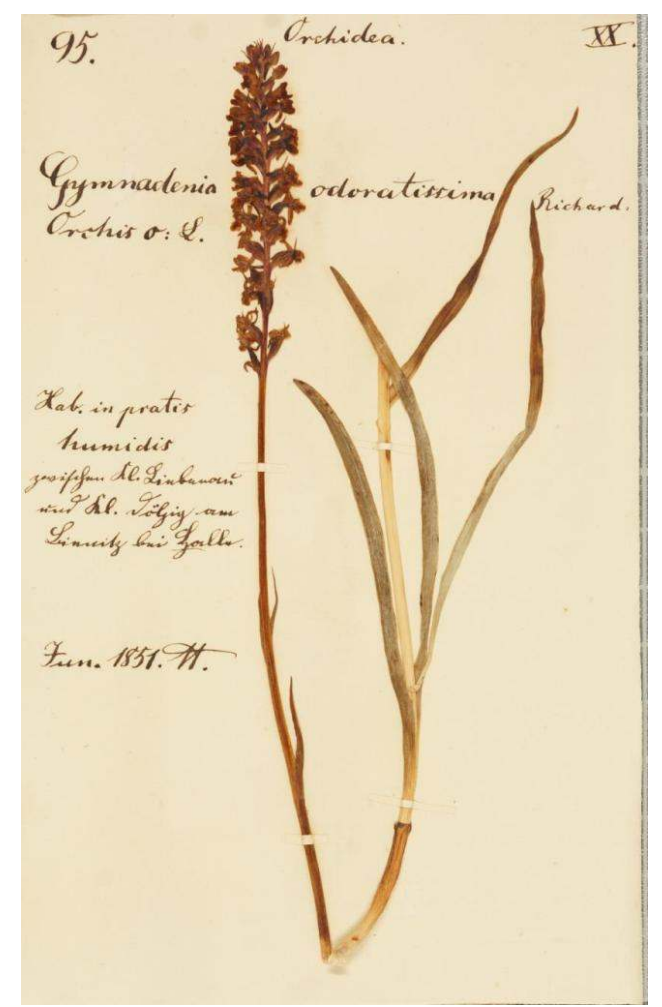

Feuillet n ${ }^{\circ} 95$ de l'herbier: Ernst Haeckel, Memorial-Herbarium, Centuria plantarum Thuringiacarum anno 1857 p. Cn. Collecta et secundum systema Linnaei sexuele distributa ab Ernesto Haeckel, AEHI, cote E 2 : «95. Orchidea. XX. Gymnadenia odoratissima Richard. Orchis o : L. Hab. In pratis humidis entre KI. Liebenau et KI. Dölzig à Bienitz près de Halle. Juin 1851. H. », AEHI, cote E 2

$\mathrm{AEHI}$

\section{L'influence de Humboldt et de Schleiden}

La dimension esthétique de la botanique avait déjà résolument imprégné le jeune Haeckel. La lecture de Die Pflanze und ihr Leben ${ }^{26}$ (La Plante et sa vie) de Matthias Schleiden constitua notamment une expérience littéraire intense pour Haeckel, et fit d'ailleurs l'objet de deux sujets de rédaction: Der ästhetische Einflu $\beta$ norddeutscher Pflanzenformationen auf den Character der Landschaft [L'influence esthétique des formations végétales du nord de l'Allemagne sur le caractère du paysage] et Föhre, Tanne und Knieholz [Pin, sapin et bois tordu $]^{27}$. Il rédigea le premier devoir en janvier 1852 alors qu'il était pris d'un fort accès de rhumatisme articulaire.

En ces temps terribles, mon unique délassement résidait dans la rédaction d'un devoir d'allemand sur l'influence des formations végétales de l'Allemagne sur le caractère du paysage, que j'effectuais avec le plus grand plaisir, et pour lequel j'étudiais l'Ästhetik der Pflanzen (L'Esthétique des plantes) de Schleiden et les Ideen zu einer Physiognomik der Gewächse (Idées pour une physionomie des plantes) de Humboldt. 28

La dissertation fit aussi l'objet d'un exposé au premier semestre à Berlin devant ses confrères étudiants du Cercle des Sciences Naturelles (Naturwissenschaftlichen Kränzchen). Haeckel envisagea également d'en faire l'exposé à Wurtzbourg, puisque le sujet lui tenait vraiment à cœur. Les Tableaux de la nature (Ansichten der Natur) d'Alexander von Humboldt 
${ }^{29}$ amenèrent Haeckel à s'intéresser à l'esthétique et cela se révèle non seulement dans la forme de ses lettres mais également dans l'aspiration à l'harmonie qui se dégage du contenu de ses lettres, de ses carnets et, en grande partie, de ses dessins et de ses herbiers. Haeckel se saisit alors de l'exigence de Humboldt concernant la représentation conforme à la nature des plantes et s'essaya à ses premières études détaillées des arbres ainsi qu'aux descriptions littéraires de plantes singulières (par exemple, le sabot de vénus). Jeune peintre paysagiste, Haeckel suivit, en outre, l'invitation de Schleiden et de Humboldt à représenter la physionomie des plantes non seulement dans leur forme spécifique, mais également au sein de leur formation végétale ${ }^{30}$.

Ainsi, Humboldt m'offrit la liste et la description de telles formations végétales qui constituent rien moins que les bases théoriques et pratiques de la physionomie végétale dont j'ai parlé et qui en fournissent la preuve pratique. En sont exclues les seules formes tropicales, dans lesquelles la puissante Nature n'a guère été altérée par la main de l'homme et se distingue de loin de toutes les autres par son abondance, sa magnificence et sa diversité. ${ }^{31}$

Haeckel entreprit alors de décrire les formations végétales de l'Allemagne du Nord d'après les exigences de Humboldt et de Schleiden. C'est ainsi qu'il jeta les fondements de son esthétique botanique auxquels il allait rester fidèle jusqu'à la fin de sa vie et grâce auxquels la zoologie allait aussi l'intéresser, par la suite, en particulier dans ses derniers récits de voyage, comme ses Lettres d'un voyageur dans l'Inde (Indische Reisebriefe) ${ }^{32}$.

Fig. 4

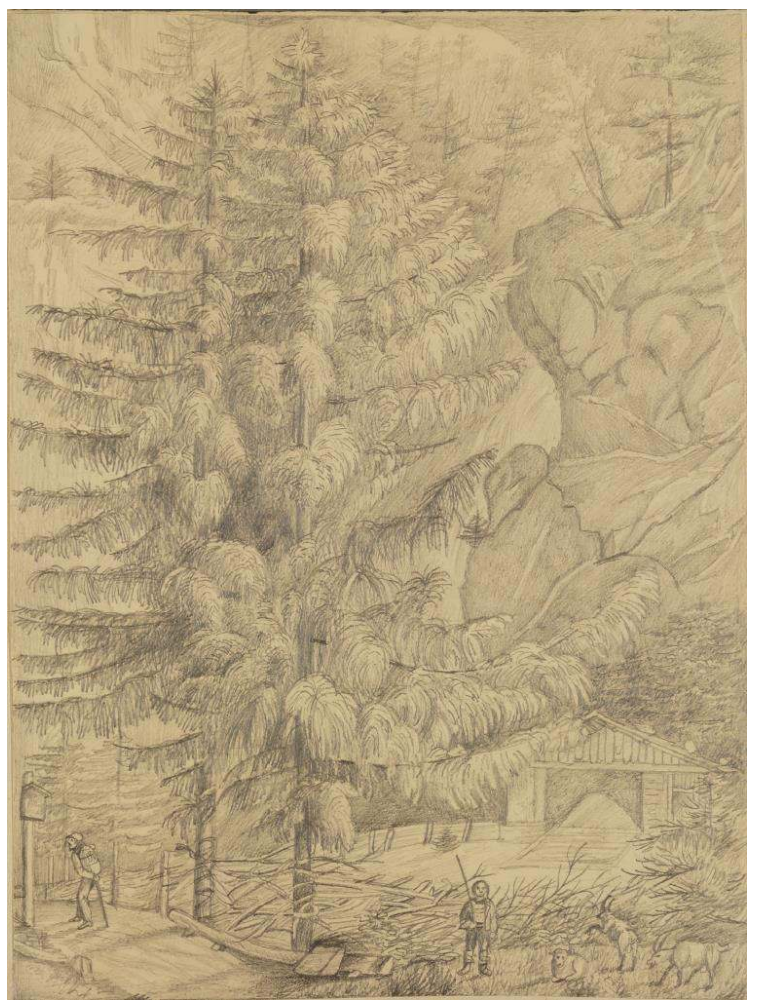

Ernst Haeckel, 20 Zeichnungen (Landschaften, Baumstudien) in Bleistift von Ernst Haeckel (Gymnasiast in Merseburg), AEHI, fol. 11

AEHI

Schleiden, en particulier, devint un inspirateur scientifique pour Haeckel. En 1850, la botanique était en voie de transformation. D'une science de la collecte et de la description 
pure, elle évoluait vers une discipline qui jouissait particulièrement de l'utilisation du microscope et des nouvelles connaissances empiriques. La botanique ne relevait, d'après Schleiden, ni d'une science inductive, ni d'une simple collection, et encore moins de la spéculation. ${ }^{33}$ Le 8 février 1851, Haeckel reçut en cadeau de la part de ses parents un microscope achromatique moderne. Il commença alors à passer toutes les plantes au microscope. Il était alors bien conscient de l'ironie avec laquelle sa "récolte de foin » entrait en contradiction avec les exigences de Schleiden. Il nota ainsi sur la couverture de son exemplaire du Synopsis der Deutschen und Schweizer Flora de Koch, deux devises opposées :

\section{Devise :}

«Le botaniste n'est pas un colporteur de noms barbares = latins, il n'est pas un homme qui cueille des fleurs, qui les nomme, les sèche et les enroule dans du papier, mais il élève toute sa sagesse dans la disposition et la classification de ces herbes collectionnées artificiellement. » Schleiden.

[et]

\section{Contre-devise :}

" Je cueille des fleurs et je ramasse du foin : Les gens appellent cela de la botanique, Mais j'ai un autre nom pour cela : timidité. » Chamisso. ${ }^{34}$

Fig. 5
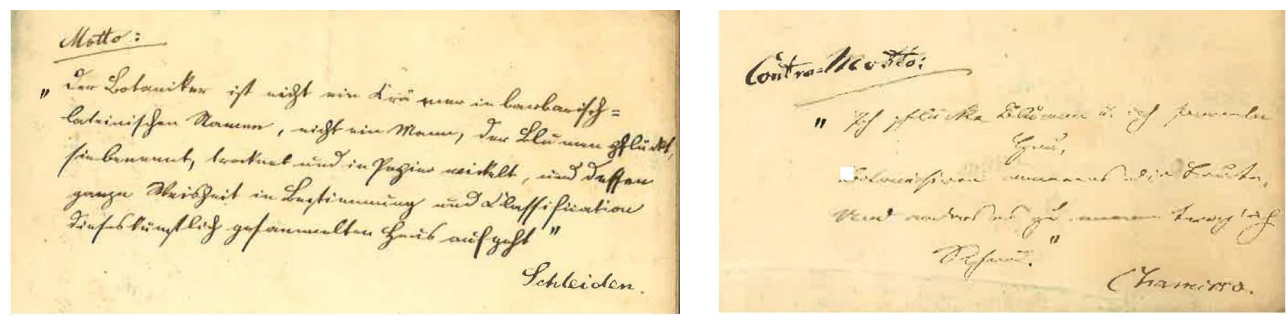

Citations de Schleiden et Chamisso dans l'exemplaire de Haeckel du Synopsis der Deutschen und Schweizer Flora de Koch

$\mathrm{AEHI}$

À partir du mois d'octobre 1851, Haeckel se vit confronté à des changements radicaux. Ses parents quittèrent leur demeure de Mersebourg et déménagèrent à Berlin. Haeckel resta en pension chez la famille Osterwald pour préparer son Abitur (baccalauréat). Bien que logé avec la famille de son professeur et trois camarades de chambre, le sensible Haeckel souffrait de la séparation d'avec ses parents. Il ne put les revoir qu'à Noël. Comme cadeau de Noël, il reçut le Pflanzenreich ${ }^{35}$ de Petermann qu'il coloria au cours des semaines qui suivirent. Un gonflement douloureux au genou droit, qui fut diagnostiqué par la suite comme la conséquence de son rhumatisme articulaire chronique, lui causa quelques problèmes après son retour de Berlin. Haeckel fut contraint de manquer les cours. Grâce à un traitement médical il put retourner en classe sept jours plus tard et finir de passer son Abitur le 12 mars avec les examens oraux. Le 13 mars, il retourna chez ses parents à Berlin et, fatigué par ses examens et impatient de reprendre ses études de botanique, il consacra son temps à

colorier le Pflanzenreich de Petermann, passer au microscope des préparations, lire Schleiden, Örsted, etc., en bref, m'adonner à toutes mes occupations préférées, librement et sans soucis! Beatus ille, qui- $!^{36}$ 
En 1852, Haeckel loua un logement à Iéna pour le semestre d'été. Rien ne semblait plus entraver l'étude tant attendue qu'il voulait faire de Schleiden. Toutefois ses douleurs au genou réapparurent et il fut alors contraint d'abandonner ses projets à Iéna, pourtant désirés depuis si longtemps, et dut rester à Berlin.

\section{Le premier semestre à Berlin en 1852}

Le 24 avril 1852, il s'inscrivit à l'Université Friedrich Wilhelms à Berlin en tant que " studiosus medicinae et naturae ». Son choix d'étudier la médecine répondait aux souhaits les plus ardents de son père. Mais en cédant effectivement aux désirs de son père et en renonçant à ses projets personnels, Haeckel plongea dans une profonde mélancolie. Le 30 mai 1852 il écrivit de nouveau dans son journal :

Ce n'est qu'aujourd'hui, dimanche de Pentecôte, que j'ai retrouvé le courage, la force et l'envie de continuer ce journal interrompu; après avoir enfin surmonté la douleur de voir anéanti tout espoir d'étudier avec Schleiden à Iéna (surtout pour les sciences naturelles), espoir pourtant caressé depuis des années. Pour autant, je n'admets toujours pas complètement la nécessité de rester à Berlin au sein de la (pourtant si chaleureuse) maison familiale (Schifferstrasse 6), où je ne trouve qu'une tranquillité apathique. ${ }^{37}$

Pendant ces premières semaines à Berlin, il dut prendre soin de sa santé et ne put prendre part aux cours que petit à petit. Néanmoins, la perspective d'étudier les sciences naturelles n'était pas, comme se l'imaginait Haeckel lors de ses périodes de doute, réduite à néant. En effet, les études de médecine exigeaient la participation à des cours de sciences naturelles. Haeckel assistait alors, parallèlement aux cours de chimie, de physique et de zoologie, à des séminaires sur la botanique dispensés par Alexander von Braun.

21 Braun donnait des cours six jours par semaine de 7 à 8 heures pour les débutants qui s'initiaient aussi bien à la botanique générale que spéciale. Haeckel assistait également aux cours de «botanique systématique » de Braun, le mercredi de 4 à 5 heures. Le samedi de 4 à 5 heures, le cours pour débutants consistait en «études botaniques ». Haeckel prenait soigneusement note de ces leçons ${ }^{38}$ même si son enthousiasme restait, au début, limité : «Imagine-toi seulement qu'ici il n'y a pas une seule personne avec laquelle je puisse converser de quoi que ce soit et notamment de botanique. $»^{39}$, écrivit-il à Weber. En raison de sa formation préalable, Haeckel trouvait trivial le contenu de ce cours, en outre il n'était pas en mesure d'éveiller le moindre intérêt pour la botanique chez ses condisciples, en tout cas pas comme autrefois avec ses trois camarades d'école. Seules les leçons de botanique réveillaient son enthousiasme :

La leçon de botanique de samedi fut la plus intéressante. Elle s'est tenue au jardin botanique et ce fut vraiment magnifique. En outre, j'ai obtenu deux ou trois fois par semaine des plantes rares, locales et étrangères, du très joli jardin botanique de l'université auquel je me suis abonné auprès de monsieur Sauer pour $5 \mathrm{rl}$ [Reichstaler] par semestre. ${ }^{40}$

Haeckel devint également plus proche de Braun, « qui était très aimable avec [lui] et qui retenait [son] nom car [il] avai[t] déjà fait de la botanique $»^{41}$. Si Haeckel avait d'abord gardé ses distances en raison des remarques désobligeantes de Braun à l'encontre de Schleiden, une relation plus amicale vit le jour au cours du semestre. Braun l'invitait à des événements privés où Haeckel pouvait rencontrer des botanistes et nouer des relations. Il 
resta en contact avec Braun durant toute sa vie, à laquelle celui-ci prit d'ailleurs part en de nombreuses circonstances.

Haeckel passa l'été avec ses parents à Teplice. Ce séjour dans cette station balnéaire était dû à son problème au genou. Un voyage initialement prévu dans les Alpes dut être ajourné en raison de sa santé ; Haeckel ne réalisa ce voyage qu'en 1855. Son temps libre était à nouveau entièrement consacré à la botanique. Haeckel collecta un nombre considérable de plantes à Teplice..$^{42}$ De retour à Berlin, il les rangea son herbier :

Durant les huit premiers jours, je ne cessai de m'occuper de mon herbier. Je dus d'abord examiner toutes les plantes que j'avais récoltées durant tout l'été pour ensuite les classer. Avec toute cette abondance de plantes, très belles et bien rangées, j'eus besoin de 12 volumes d'environ un pied d'épaisseur.

$[\ldots]$

Enfin, je rendis une nouvelle fois visite au Dr. A. Garcke, au 104 de la rue de Potsdam, qui sembla enchanté de ce que je lui communiquai sur la flore de Teplice.

Il m'offrit alors certaines plantes rares de la Flora Hallensis. ${ }^{43}$

Bien qu'il souffrît encore de la déception provoquée par l'évanouissement de ses projets à Iéna, il put consacrer une part considérable de son temps à la botanique pendant ce premier semestre. Le semestre suivant fut consacré aux cours de médecine. Pour continuer ses études, Haeckel devait choisir entre Göttingen, Iéna et Wurtzbourg. Il se décida pour Wurtzbourg car la faculté de médecine s'y distinguait des autres par sa très bonne réputation.

\section{2-1853 : le premier semestre à Wurtzbourg}

À Wurtzbourg, après les premières dissections de cadavres, Haeckel se rendit à l'évidence : la médecine ne pouvait pas devenir sa profession. Son dégoût des maladies, sa propre hypocondrie ainsi que la faiblesse de ses nerfs lui compliquèrent particulièrement la tâche en ce début de semestre. Il continua tout de même à assister aux cours d'anatomie humaine de Albert von Kölliker ${ }^{44}$ et poursuivit les dissections. Il appréhendait alors cela « du pur point de vue de l'histoire naturelle (non médicale !), en tant qu'histoire de la nature humaine $»^{45}$. Sans pour autant dissiper ses doutes et ses réticences, il fut assez convaincu par la personnalité de Kölliker et par ses cours : "L'anatomie en ellemême est, comme je l'ai déjà dit, merveilleuse, - mais uniquement si l'on met de côté les pathologies et l'histoire de la maladie ! ${ }^{46}$ Un changement de spécialité se dessinait alors. Pourquoi ne pourrait-il pas faire comme ses amis du «trèfle botanique » et étudier les sciences naturelles et les mathématiques? Les parents de Haeckel prirent au sérieux les soucis de leur fils, mais lui recommandèrent néanmoins de poursuivre les études dans lesquelles il s'était engagé.

En compensation, Haeckel se tourna de nouveau vers la botanique. À l'occasion d'une conférence au sein de la Société physico-médicale, il fit la connaissance du botaniste August Schenk ${ }^{47}$ et assista alors à ses séminaires :

Cette semaine ont aussi commencé les cours pratiques chez le professeur Schenk: "Études microscopiques des tissus végétaux ». Je me réjouis particulièrement de cette belle rencontre. À part moi, deux personnes seulement prennent part à ce cours, j'ai donc la chance de fréquenter un véritable botaniste de renom, ce qui était mon souhait le plus cher depuis si longtemps. Les cours ont lieu le mardi et le jeudi, de 6 à 8 heures du soir. Nous y disposons chacun d'un beau microscope avec lequel nous pouvons observer les superbes préparations qu'il réalise. Nous les 
dessinons, il nous les explique et nous pouvons lui poser toutes les questions que l'on souhaite, tant qu'elles restent claires. Vous pouvez vous imaginer quelle joie cela me procure et tout ce que cela représente pour moi. ${ }^{48}$

Haeckel établit vite le lien avec Schenk et sa famille. Mais les rapports n'étaient pas toujours simples car Haeckel ne partageait pas les opinions politiques de Schenk, ce qui donna lieu à quelques discussions tumultueuses. Malgré leurs quelques différends, les rapports entre Schenk et Haeckel restaient cordiaux. Un soir de 1852, Schenk le présenta même à son cercle d'amis. Une lettre du $1^{\text {er }}$ janvier 1853 dresse un portrait très riche en explications du rapport qu'entretenait Haeckel avec la botanique. Il y peint très clairement sa position à ses parents. Haeckel se trouvait au carrefour des disciplines :

Pour autant que je puisse en juger, la médecine ne se trouve que dans un coin, cachée derrière l'arbre. «L'arbre doré de la vie » est et reste la botanique ! [...] Devant lui se trouve sur la table une pile galvanique, un aimant, une pince, des lamelles, des tubes à essai, ainsi que le matériel commun à toutes les sciences naturelles. Dans le fond à gauche se trouve une perspective d'avenir effrayante, un tableau noir sur lequel est écrite une interminable formule mathématique qui reste encore à résoudre. Au premier plan, l'Atlas physique de Berghaus, qui constitue bien maintenant le tout premier plan d'Ernst Haeckel lui-même !49

Fig. 6.

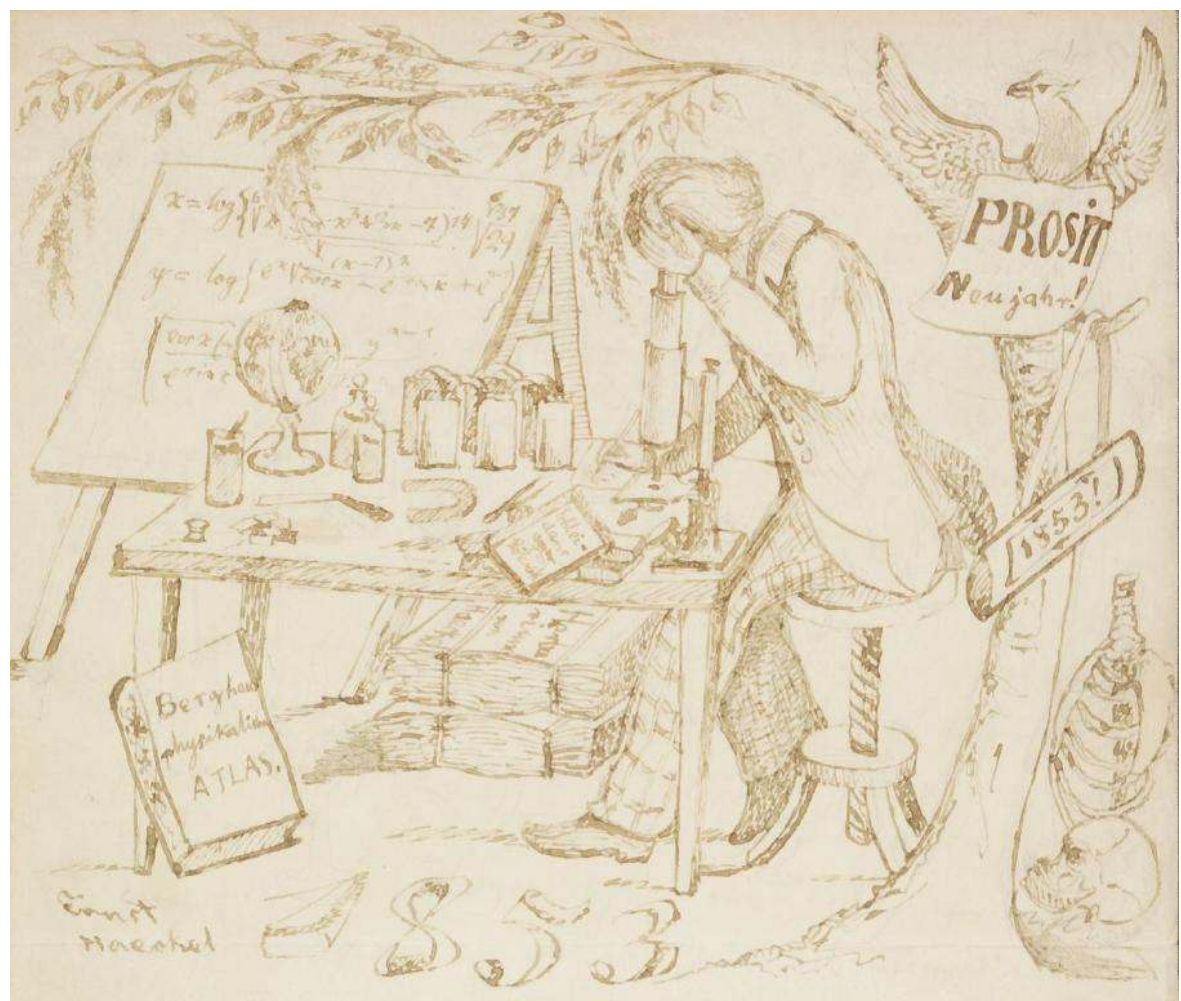

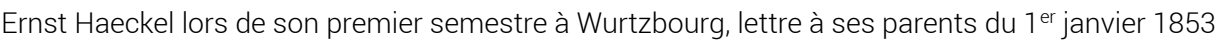

Devant le microscope se trouve un ouvrage botanique de Schleiden, une boîte de botaniste est accrochée à l'arbre, en dessous de la table sont empilés deux forts dossiers cartonnés contenant des plantes séchées. Haeckel est au microscope. Ce sont surtout les cryptogames qui mobilisaient son attention. Il empruntait des livres spécialisés de botanique dans la bibliothèque de Schenk et ce dernier lui donna même quelques plantes rares. Avec les cryptogames, il commençait à se pencher, sous l'égide de Schenk, sur une 
famille de plantes qui l'intéressait non seulement par sa systématique, mais aussi par la possibilité qu'elle offrait d'être observée au microscope. Le développement des fougères et des mousses le fascinait particulièrement. Une porte s'ouvrit alors sur les questions actuelles de la botanique scientifique, comme celles que Schleiden exposait dans ses "grandes orientations" („Grundzügen“) et qui se positionnaient aux antipodes de la simple «récolte de foin ». À partir de là, Haeckel aurait pu devenir botaniste professionnel. Mais Schenk ne souhaitait pas que Haeckel s'accroche à lui. Schenk, en effet, n'avait pas l'âme d'un mentor, que ce soit sur le plan scientifique ou personnel. À côté des observations au microscope, la « récolte de foin » n'était cependant pas négligée. Haeckel écrivit fièrement à son père :

Cher papa, peux-tu de nouveau déménager les meubles de ma grande pièce (je veux dire, au cas où tu l'aies utilisée) car une belle meule de foin d'un pied de diamètre va venir enrichir ma grange d'une très belle manière. La grande table retrouvera ainsi sa place dans ma grange, couverte de plantes et comblée de foin, pour mon plus grand plaisir ! $!^{50}$

Haeckel restait l'incorrigible «homme des plantes» („Pflanzenmensch“). Les cours prirent fin à la mi-mars. Et à la mi-avril, il retourna de nouveau à Wurtzbourg mais non sans rendre visite à ses amis botanistes à Halle et sans entreprendre avec eux les premières excursions du printemps. Pour obtenir quelques conseils sur ses études futures, il rendit également visite au directeur du jardin botanique de Halle. Mais la rencontre entre Haeckel et Schlechtendahl ${ }^{51}$ ne fut pas des plus plaisantes :

Je suis donc allé chez Schlechtendahl et n'ai pas tiré grand-chose de notre conversation, notamment sur le chapitre professionnel. Il m'a conseillé d'abandonner la botanique (puisque malgré toute la volonté du monde, je n'en aurais pas la force nécessaire); cependant, j'ai appris plus tard par Henkel qu'il concevait sa relation avec la jeune génération d'une manière très égoïste, qu'il ne cherchait pas à la soutenir, mais qu'il essayait au contraire de la détourner de la botanique. Je ne m'en soucie guère $!^{52}$

À Mersebourg, Haeckel rendit visite à ses vieux amis et à ses professeurs qui eux, au contraire, le soutinrent dans les ambitions qu'il nourrissait au sujet de la botanique :

Je me suis donc rendu chez Lüben, qui m'a reçu très aimablement et chez qui je suis resté plus de quatre heures, durant lesquelles nous nous sommes mutuellement confié le fond de nos pensées naturalistes et botaniques en particulier. Nous avions, en effet, les expériences de toute une année à partager ! Il pensait, comme tous les autres amis dont je parle (notamment Weiß, qui fut le premier à soutenir cela, ainsi que Osterwald et tous les autres), que je ne pourrais, à vrai dire, rien faire d'autre, mais que je ne pourrais également rien faire d'aussi bien que professor botanices!ô! ô! ô!- Ô scientia amabilis; Quando tandem tecum in aeternum conjungas ?!!!53

31 Haeckel était perdu. Tandis que ses parents et son frère lui conseillaient de continuer ses études de médecine, ses vieux amis l'encourageaient à la botanique.

\section{Le deuxième semestre 1853 à Wurtzbourg}

Après son arrivée à Wurtzbourg, il rendit visite à un collègue de Schenk spécialisé en botanique médicinale, sans pour autant en attendre quelque chose de particulier :

À 8 heures, j'étais au cours de botanique médicinale de Schenk qui à vrai dire (mises à part les belles plantes que l'on a pu y voir) m'a semblé pour le moins inutile. J'y ai cependant poliment assisté, plus par égard pour lui que pour moi-même, car il me l'avait vaguement proposé. Je reste, tout du moins, en bons termes avec lui. Mais je 
n'ai vraiment pas de chance avec les cours de botanique car je n'ai encore rien entendu dont je sois vraiment content. ${ }^{54}$

Haeckel ne voulait pas dégrader sa relation avec Schenk, et ainsi continuer de recevoir de sa part des plantes pour son herbier, mais il renonça à suivre son cours dès la semaine suivante :

Je ne me suis pas présenté, à mon grand profit et à son grand agacement, au cours de botanique médicinale de Schenk auquel j'avais assisté la semaine passée. [...] Je suis donc allé voir Schenk et lui ai annoncé que la volonté de mon père, ainsi que ma propre conviction, me commandaient de devenir médecin, et que pour cela, je devais me préparer cet été. En conséquence, je ne pourrais pas m'occuper de ce fourbi de plantes inutiles, ce à quoi Schenk répondit que mon père avait bien entendu raison, mais que s'il me laissait étudier la médecine je devais donc bien étudier la botanique médicinale. ${ }^{55}$

Les hautes ambitions de Haeckel, ses études de médecine ainsi que la volonté de son père constituèrent des prétextes suffisants. Grâce à cela, Haeckel put se tirer d'affaire. Cependant, les véritables raisons étaient d'un ordre bien plus profond. Schenk n'était pas au niveau des attentes de Haeckel. Ses cours étaient pénibles à suivre et Haeckel était maintenant bien trop avancé dans cette discipline pour y apprendre quoi que ce soit. Après sa mauvaise humeur du début - «Je suis également tombé en disgrâce auprès de Schenk, parce que je n'ai pas eu la patience de suivre son cours ennuyeux $\|^{56}$ - Haeckel s'excusa et l'invita à se joindre à lui pour des excursions botaniques dans la riche nature de Wurztbourg. Haeckel passa ainsi tout l'été à collecter une fois de plus tout un « fourbi de plantes inutiles » :

Dans une forêt, près d'un ruisseau, nous étions en quête de la plus belle orchidée allemande, la magnifique Cypripedium Calceolus ; et j'eus la chance de trouver deux spécimens de cette belle et rare espèce sur les quatre que nous avons pu trouver. Une très grande et unique fleur, ou deux tout au plus, trône au bout d'une fine tige feuillue. Quatre pétales, étroits et ondulés, arborent un beau violet foncé et sont disposés face à face en forme de croix (a dans le croquis). Au centre de ceux-ci vient se loger un cinquième petit pétale, le plus souvent de la forme d'une barque (c), et, en dessous de ce dernier, surgit un sixième pétale très grand (b), arborant un magnifique j'aune d'or, et qui prend la forme creuse d'un sabot ou d'une barque ventrue. C'est pour cette raison que cette splendide plante s'appelle aussi le «Sabot de Vénus" [„Frauenschuh"]. Vous pouvez à peine vous imaginer comme je me suis réjoui de cette trouvaille tant espérée. ${ }^{57}$ 
Fig. $7 a$

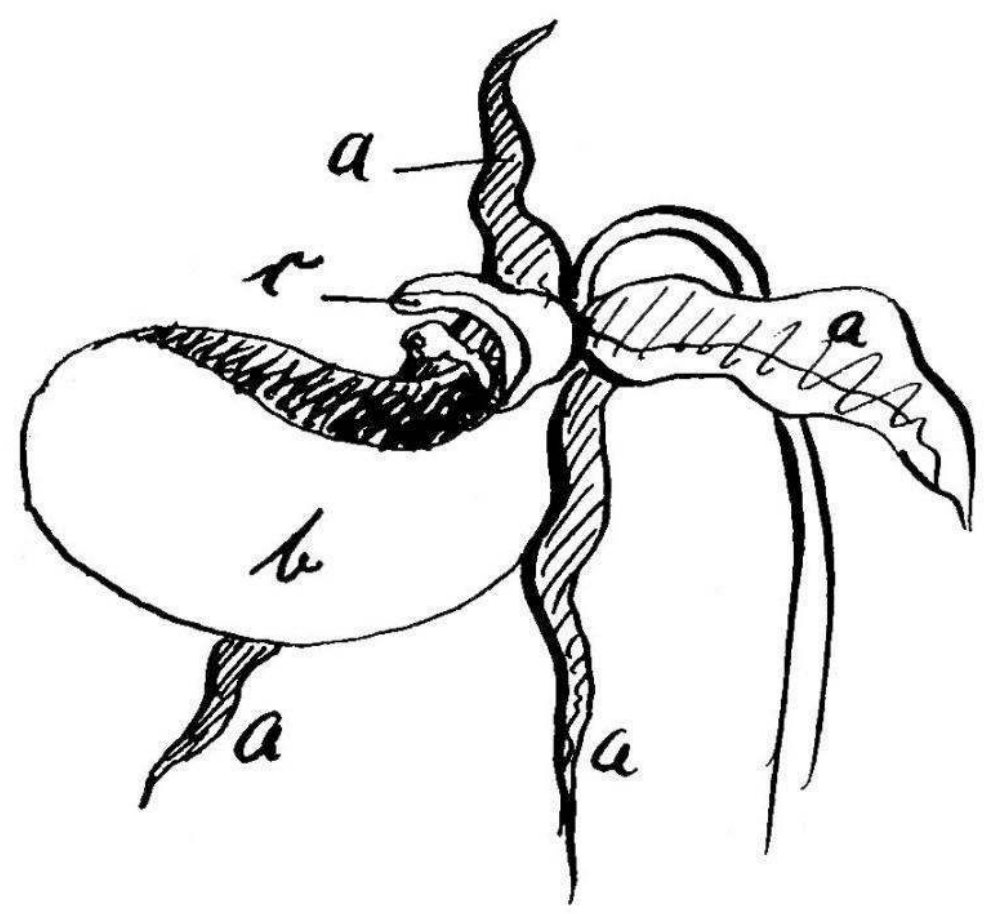

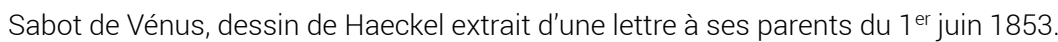


Fig. 7b

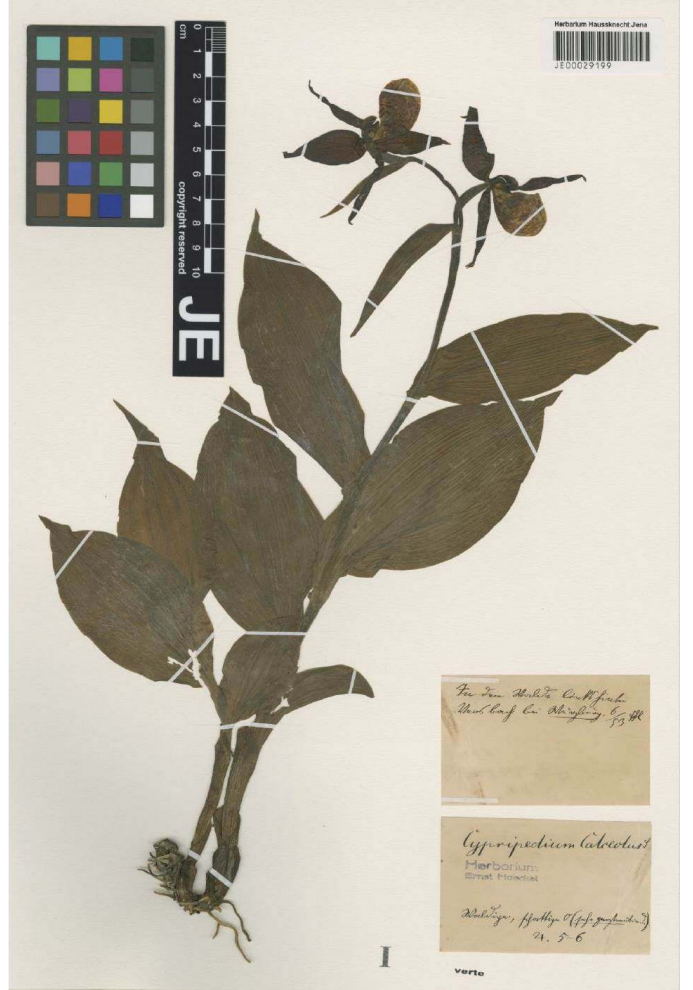

Cypripedium calceolus L., spécimen de l'herbier de Haeckel (Herbarium Haussknecht Jena, JE 000229199) : " Dans les bois, à gauche derrière Moosbach près de Würzburg. 6/53 HI. » ;

«Cypripedium Calceolus L Waldige, lieux ombragés (peu fréquent en Allemagne) ㄴ.1. 5-6 ».

Sur le plan scientifique, Haeckel était surtout intéressé par le cours de physiologie de Kölliker qui lui offrait au moins une approche théorique de la médecine, «si bien qu'en fin de compte [il] gard[ait] quand même un peu de courage à la pensée qu['il] deviendrai [t] medico-botanicus ou botanico-medicus, ce qu['il] daignerai[ $t$ ] rester, mais plus comme "homme des plantes" que comme "homme des hommes" $»^{58}$. Néanmoins, une expérience déterminante pour lui fut un cours de Kölliker sur l'anatomie comparée qui était en réalité un cours de zoologie. Il trouva en Kölliker - tout du moins au début - un scientifique qui lui offrit beaucoup, aussi bien sur le plan personnel que professionnel et esthétique. Comme les cryptogames qui le fascinaient autrefois, ce fut au tour des animaux marins de le captiver :

Ce n'est qu'aujourd'hui que je vous écris de nouveau, car les merveilleux polypes, méduses, coraux et tant d'autres, m'ont occupé toute la semaine passée, de 5 heures du matin à 10 heures du soir, et ce pour mon plus grand plaisir. Désormais, ma passion pour la zoologie qui, enfant déjà, avait attiré mon attention avant même l'histoire naturelle et qui constituait une de mes occupations préférées, s'est à nouveau vivement ranimée et m'a ouvert, grâce aux connaissances sur l'anatomie que j'ai acquises, le merveilleux chemin vers l'étude des animaux. ${ }^{59}$

Haeckel gardait des polypes d'eau douce dans sa chambre d'étudiant, mais ceux-ci ne constituaient alors qu'un faible ersatz des voyages en milieu marin auxquels il aspirait :

Kölliker a maintenant passé en revue les étoiles de mer et les oursins, et j'ai tout à coup eu une terrible envie d'aller en mer pour observer ces magnifiques espèces et pour découvrir leurs splendides formes artistiques en milieu naturel. C'est tout de 
même bien autre chose que les illustrations, même si celles-ci sont très belles. D'ailleurs j'en ai déjà des centaines dans mon carnet. ${ }^{60}$

Cet été, il continua certes d'agrandir continument sa collection de plantes, mais son intérêt croissant pour la zoologie apparut très nettement dans ses lettres :

J'ai d'ailleurs rassemblé maintenant un beau paquet de végétaux, ce qui correspond à environ quatre volumes de mon herbier, une partie provenant de la Flora Herbipolitana, une autre partie provenant du jardin botanique d'ici, et je nourris toujours des craintes quant à leur acheminement jusqu'à Berlin! - Mais ces réjouissances botaniques sont maintenant en partie suppléées par la zoologie. Par ce biais je comprends l'anatomie comparative, laquelle est véritablement une science unique! Nous faisons à présent d'habiles dissections d'escargots, de coquillages, etc. ${ }^{61}$

En juillet, sa première étude scientifique vit le jour sous la direction de Kölliker: Skizzen aus der Entwicklungsgeschichte der Maulwurfsgrille Gryllothalpa vulgaris ${ }^{62}$.

Koelliker et moi sommes à présent assez proches. La semaine passée, je lui ai apporté des œufs de courtilière (Gryllotalpa) que j'avais ramassés au jardin botanique. Il m'a prié d'examiner le développement du jeune insecte dans l'œuf, et m'a, en outre, mis à disposition un microscope avec lequel je peux travailler à tout moment dans la salle d'anatomie. C'est ainsi ce qui m'occupe quelques heures chaque matin. Leur développement est extrêmement intéressant et pourrait mener à de véritables résultats; si seulement je n'étais pas si terriblement maladroit, notamment pour les préparations ! 63

Durant les vacances, Haeckel dut une nouvelle fois s'occuper de son genou malade et partit en cure avec ses parents à Rehme en Westphalie. Le nouveau microscope ${ }^{64}$ qu'il $^{\prime}$ reçut en cadeau lui procura une joie toute particulière, puisqu'il en avait depuis longtemps demandé un à ses parents. Il consacrait ainsi la majeure partie de son temps à utiliser son microscope. Loin de la mer, il examinait de nouveau des cryptogames. Aussi, lors de la visite qui suivit à Ziegenrück, Haeckel n'avait-il d'yeux que pour les cryptogames et son nouveau microscope - son « Schieck ».

Ainsi quand le Dr. me trouva hier profondément absorbé dans l'étude des mousses, il transforma ainsi le verset de la Genèse: «Et Dieu dit: Il n'est pas bon que l'homme soit seul; et il créa pour lui des Bryophyta, des Hepaticophyta et des Anthocerotophyta, et un microscope $! »^{65}$

\section{Le troisième semestre à Wurtzbourg. Vivat Microscopia!}

Le semestre de l'hiver 1853-1854 fut entièrement placé sous le signe de la médecine et fut consacré à un travail intense. Durant cette période, Haeckel dut mettre un peu de côté sa collection de mousses. Les cours de Virchow sur la pathologie cellulaire l'intéressaient particulièrement, mais ils ravivaient par la même occasion ses réticences passées à l'égard de la médecine. Ces cours s'articulaient souvent autour de l'obstétrique et d'essais chimiques en laboratoire. Il appréhendait avec angoisse la perspective de faire de la médecine sa profession. Il exposait de plus en plus ses doutes dans les longues lettres qu'il adressait à son père et rêvait d'une vie de voyageur avec son microscope :

Combien des hommes les plus brillants pratiquent cette merveilleuse discipline! C'est grâce à Schwann, Schleiden, Koelliker, Virchow et Schacht que la plus belle de toutes les sciences acquiert toute sa gloire. Combien des hommes les plus brillants de cette époque se livrent maintenant en tous lieux aux observations microscopiques les plus précises et les plus minutieuses !66 
Parfois même, ces perspectives d'avenir prenaient un aspect quelque peu saugrenu :

Mon unique consolation réside alors dans l'idée folle de me retirer, dans le pire des cas, avec mon microscope que je ne lâche plus, dans n'importe quelle forêt vierge de la Guyane et d'étudier la nature à ma guise. ${ }^{67}$

Il signa cette lettre « L'Oncle, Dr. en phil. et med. Professeur associé en Microscopie » ${ }^{68}$. Il projetait tous ses rêves sur son neveu ${ }^{69}$, tout juste né, et le voyait

bientôt comme un joyeux petit garçon grandissant dans la montagne aux forêts magnifiques de Ziegenrück jusqu’à devenir étudiant en microscopie, en botanique, en anatomie animale, etc., et enfin jusqu'à devenir un naturaliste voyageur (à Borneo, à Madagascar, en Nouvelle-Guinée, au Brésil ou bien où tu voudras) [...] Ceci reste mon rêve de toujours et de devoir y renoncer me coûte les plus cruels efforts. J'ose espérer que mon neveu puisse un jour remplir cette merveilleuse mission ! ${ }^{70}$

Le jeune " professeur associé en microscopie » fit néanmoins une communication au sein du Cercle de médecine et des sciences naturelles (Medizinisch-Naturwissenschaftliches Kränzchen) de Kölliker. Il voulut d'abord présenter, comme il l'avait déjà fait à Berlin, un exposé portant sur la géographie des plantes. Puis il se décida pour un exposé sur la «reproduction des plantes cryptogames ». Haeckel emprunta à Schenk les Vergleichende Untersuchungen de Hofmeister et prépara très minutieusement sa présentation ${ }^{71}$. À l'occasion de ce travail, il redevint entièrement botaniste. Dans une longue lettre, il exposa à son père ses projets futurs de botaniste sous les tropiques. Il projetait, entre autres, d'étudier la médecine d'arrache-pied afin de trouver l'argent nécessaire pour un voyage jusqu'aux pays tropicaux en travaillant comme médecin sur les bateaux. Avec son " épouse », c'est-à-dire avec son microscope, il serait facile de survivre puisque...

On trouve dans la forêt vierge de quoi se nourrir suffisamment (car un seul petit pied de bananier suffit à nourrir un seul homme); et en cas de besoin, je pourrais me procurer le nécessaire par quelques boniments en tant que médecin (!), chirurgien (!!) et obstétricien (!!!) auprès des Indiens. Je disposerais alors de quelques années pour me nourrir à satiété - ventre et esprit! - des délicieuses faune et flore tropicales, et je tenterais ensuite de rentrer de la même manière que je serais venu, soit pour prendre une place de professeur d'université soit pour gagner, de ma plume, une maigre pitance $!^{72}$

Les parents se rendaient progressivement compte que leur fils n'envisageait pas vraiment de devenir médecin. Cependant, finir ses études de médecine restait une condition sine qua non pour réaliser ce voyage dans les tropiques tant désiré. C'est pourquoi Haeckel continua ses études de médecine pendant le semestre d'été de 1854 à l'université de Berlin. Ce semestre eut une importance considérable, à de multiples égards, pour l'intérêt qu'il manifesta pour la zoologie.

\section{Berlin 1854}

Haeckel travailla beaucoup et suivit les cours afin de se préparer à l'examen intermédiaire de médecine, le tentamen philosophicum:

Cet été amorça la transformation qui se produisit en moi l'hiver qui suivit. Il est vrai que le semestre d'été ne fut pas particulièrement agréable. J'étais tellement surchargé de cours (la plupart de philosophie) (chaque jour de 7 à 14 heures) que je ne disposais presque d'aucun temps libre $[. . .]^{73}$ 
Les séminaires de Johannes Müller ${ }^{74}$ l'enthousiasmaient particulièrement. En Müller il trouva enfin son mentor spirituel et scientifique. Pendant cet été à Berlin, son intérêt se tourna définitivement, sous l'influence de Müller, vers la zoologie :

Il m'a apporté énormément à tous points de vue, et tout particulièrement par ses cours magistraux auxquels j'ai voué une véritable adoration (en physiologie et surtout en anatomie comparée). J'ai fait connaissance ici pour la première fois avec une figure d'autorité reconnue par tous, qui tenait dans mon estime le rang d'idéal scientifique, et dont les activités spécialisées (au Muséum, etc.) m'amenèrent à considérer pour toujours l'anatomie comparée comme ma science préférée. ${ }^{75}$

Haeckel suivit scrupuleusement les cours de Müller. Au muséum, il étudiait et dessinait des préparations anatomiques et entrait personnellement en contact avec Müller. Celui-ci ne fut pas seulement le nouveau mentor de Haeckel mais l'astre autour duquel gravitait toute une génération d'ambitieux scientifiques. Ainsi, Kölliker, Virchow et Gegenbaur ont soit fait leurs études auprès de Müller, soit eu avec lui une collaboration scientifique. De ce fait, Haeckel faisait partie d'un vaste réseau ${ }^{76}$. La botanique fut désormais remisée au second plan, derrière la zoologie. Cependant, Haeckel continuait de se rendre certains soirs chez Alexander Braun pour rencontrer des chercheurs et, avant tout, des explorateurs. Son objectif était, en définitive, de faire un voyage de recherche dans les tropiques, désormais à la fois en tant que zoologiste et botaniste.

\section{Heligoland : le choix de la zoologie}

Entre le 17 août et le 17 septembre 1854, un rêve se réalisa pour Haeckel. Avec son camarade d'étude La Valette ${ }^{77}$, il entreprit son premier voyage en mer pour l'île d'Heligoland en Mer du Nord. Ce qu'il n'avait pu observer jusqu'à présent qu'aux cours de Kölliker et de Müller ou bien dans les livres qu'il coloriait soigneusement, il l'avait là, vivant, devant ses yeux. Durant les deux premières semaines de son séjour, il récolta un grand nombre d'espèces marines. Du côté des plantes, il n'y avait sur l'île, à part les algues, que peu de découvertes à faire ${ }^{78}$. Mais la faune marine se manifestait autour de Haeckel avec une telle abondance qu'il en tira, avec ivresse, les bases de sa collection zoologique $^{79}$. Il écrivit plein d'enthousiasme à ses parents :

Je ne peux pas vous écrire ceci comme je le voudrais et je vous l'expliquerai de vive voix; une chose seulement: ma décision de devenir naturaliste, c'est-à-dire d'étudier la faune des côtes tropicales, est maintenant prise (il est plus simple, en l'occurrence, de prendre une décision humaine sans l'approbation divine!) et la zoologie a définitivement, et pour toujours, pris la place de la botanique dans mon cœur. ${ }^{80}$

Après deux semaines, Johannes Müller et son fils Max $^{81}$ vinrent sur l'île pour y effectuer des recherches. La Valette et Haeckel prenaient désormais véritablement leur place au sein d'une équipe de recherche zoologique :

Il n'est plus question de récolte, de séchage, de classement et de dissection ; au lieu de cela, chaque jour, très tôt, nous sortons en mer avec les deux Mueller, pendant une à deux heures, et nous y attrapons en peu de temps des milliers de créatures marines des plus captivantes à l'aide d'un filet. La plupart de celles que nous prenons sont des invertébrés, des radiolaires, des vers et des crustacés dont nous pouvons ensuite observer au microscope les stades de développement. Nous passons tout notre temps libre à cela et il nous faut d'ailleurs beaucoup, beaucoup de temps avant d'en venir à bout. ${ }^{82}$ 
Lors de son séjour à Heligoland, Haeckel se tourna définitivement vers la zoologie et ce de différentes manières. Tout d'abord, il commença par accroître son activité de collecte de spécimens zoologiques. Il trouva, ensuite, sous la direction de Müller sa voie dans la recherche active. Et enfin, il fut tout simplement émerveillé par l'esthétique de la faune marine :

Ma première expédition scientifique eut lieu pendant les vacances d'automne en 1854 (17-17 septembre) et fut un séjour de cinq semaines à Heligoland en compagnie de J.Mueller, lors duquel je retirai non seulement une grande satisfaction des relations que j'entretenais avec chacun (voir à ce sujet les lettres à mes parents), mais dont je revins également riche de connaissances et de trésors (faune marine, fucoïdes, etc.) ; cette nouvelle vie, que j'appris à connaître et à apprécier grâce à ce formidable voyage en mer, élargit mes perspectives de manière exceptionnelle et me donna avant tout une direction claire et un véritable but dans la vie. À cette époque, je pris la ferme résolution (qui m'avait semblé être un vœu pieux quand j'étais enfant) de me donner pour plus grand objectif de ma vie de faire un voyage à visée scientifique, botanique et zoologique dans les pays tropicaux. Cette nouvelle perspective fit disparaître comme par enchantement toutes mes idées noires et ma mélancolie. Une nouvelle vie pleine d'espoir et de courage s'offrait à moi. Je revins de Heligoland à Berlin plus sain que jamais de corps et d'esprit, comme après une renaissance. J'étais changé au plus profond de mon être. 83

\section{La botanique après 1854}

Avec cet été passé à Heligoland, Haeckel avait définitivement choisi de faire de la zoologie son métier. Johannes Müller devait toujours rester son mentor scientifique, même après sa mort en 1858. Cependant, Haeckel ne cessa pas pour autant de s'occuper de ses plantes. D'août à septembre 1855, il put enfin effectuer le voyage dans les Alpes qu'il avait tant désiré. Il fit une énorme récolte de plantes. Et de son voyage de recherche à Villefranchesur-Mer près de Nice pendant l'été 1856 , il ne retira pas que des résultats en zoologie pour sa thèse toujours en suspens. Haeckel se livra à une intense récolte de flore méditerranéenne et prit contact avec les botanistes locaux pour échanger des plantes rares. Enfin, durant son semestre de clinique à Vienne près du Rax en 1857, ses excursions botaniques furent autant d'occasions pour Haeckel de profiter du Lac de Neusiedl et des environs de Vienne pour y récolter des plantes printanières alpines. Même durant son grand voyage en Italie de 1859 à 1860, il ne cessa bien évidemment pas de récolter des plantes. Mais, prenant la place des plantes dans son esprit, les objets qui l'occupaient de plus en plus, et dont il traiterait dans ses grandes monographies, étaient ceux de la zoologie.

En 1860, il se porta candidat à un poste de professeur au lycée académique de Hambourg ${ }^{84}$ . Haeckel devait seulement prouver son expertise aussi bien en botanique qu'en zoologie. Il demanda donc à Alexander von Braun de rédiger une lettre de recommandation garantissant sa compétence en botanique. Braun accepta et écrivit ceci à propos de Haeckel :

Je souhaiterais vivement que le Dr. Haeckel, si votre choix porte sur sa candidature, puisse se concentrer sur sa discipline principale, la zoologie, bien qu'il ne puisse pas défaillir non plus à la tâche si celle-ci portait sur la botanique dont il a une connaissance approfondie. Haeckel fut naguère un de mes élèves les plus impliqués et appliqués ainsi qu'un de mes compagnons d'excursion botanique, et depuis il n'a cessé de suivre les progrès essentiels de la science botanique et prête toujours une 
vive attention au monde des plantes lors de ses voyages lors desquels il profite de

chaque occasion pour récolter quelques spécimens. ${ }^{85}$ chance d'exercer un emploi académique dans la botanique.

\section{Haeckel botaniste : une facette encore méconnue de sa biographie}

Contrairement à son succès en zoologie, Haeckel n'a jamais excellé dans ses travaux scientifiques en botanique. Si l'on souhaite étudier le rapport que Haeckel entretenait avec la botanique, c'est un point à ne pas négliger. En effet, son expertise n'atteignit pas le niveau d'un travail scientifique à proprement parler.

On aurait pu s'attendre à une monographie en botanique générale ou spéciale, comme il en produisit en zoologie. Haeckel possédait les aptitudes nécessaires dans les deux domaines. Il pouvait parfaitement trouver de l'intérêt à une monographie traitant d'un type de mousse comme à des études microscopiques de plantes cryptogames. Mais il lui manquait surtout un mentor scientifique du rang de Johannes Müller. L'admiration initiale qu'il vouait à Schleiden avait été éclipsée par les mérites du grand zoologiste. Braun et Schenk n'exercèrent pas sur Haeckel une grande influence, alors qu'il aurait eu besoin au cours de ces premières années d'une telle personnalité. On comprend bien pourquoi, à la lecture de sa correspondance. Haeckel n'était pas sûr de lui et son incertitude le menait souvent jusqu'au doute maniaque. À ses débuts, celui qui allait devenir le champion de l'évolutionnisme avait toujours besoin qu'on l'éclaire. Johannes Müller, et plus tard Charles Darwin, répondaient à cette attente.

Une autre raison qui poussa Haeckel à se tourner vers la zoologie tient à son environnement scientifique. À Berlin et aussi à Wurtzbourg, Haeckel était entouré d'une génération de jeunes scientifiques qui le menait aux questions actuelles de la zoologie. Enfin, la dimension esthétique de la faune marine est également un facteur d'influence à ne pas sous-estimer dans la décision de Haeckel. Il avait déjà succombé à son charme au cours de Kölliker et sa passion pour la zoologie naquit définitivement à son premier contact avec la faune marine à Heligoland.

57 Le plus grand héritage des travaux botaniques de Haeckel réside dans l'herbier, dont il tira un petit nombre de Memorial-Herbaria qui se trouvent aujourd'hui aux Archives Ernst Haeckel à Iéna. Il offrit son «Grand Herbier » („Große Herbarium“) en juin 1912 à l'Herbarium-Haussknecht de Weimar (aujourd'hui à Iéna) qui l'intégra à ses collections avec l'approbation de Haeckel. Sa propre collection de 12000 plantes comptait une multitude de spécimens rares parmi lesquels Joseph Bornmüller, alors conservateur de l'Herbarium, remarquait particulièrement les cryptogames et les espèces qu'il considérait comme des documents sur la flore méditerranéenne :

Cette collection a tout d'abord une haute valeur historique et conserve à ce titre son originalité. Elle deviendra ainsi une pièce maîtresse de notre institution, que j'ai l'honneur de diriger. ${ }^{86}$

En 1854, Haeckel renonça complètement à ses ambitions scientifiques en matière de botanique. Mais ses travaux sur les plantes s'étaient développés, depuis ses premiers essais, dans différentes directions. Outre sa collection et la botanique en tant que science académique, les plantes exerçaient un fort attrait esthétique sur Haeckel qui a cherché 
d'une part à en donner une traduction littéraire dans ses récits de voyage et ses lettres, d'autre part à le restituer par le dessin, l'aquarelle et plus tard la peinture à l'huile. Ce dont Haeckel avait si fort rêvé dans sa jeunesse resta longtemps hors de portée : le voyage dans les tropiques. Ce n'est qu'en 1881, et plus tard en 1900, que deux voyages le menèrent jusqu'à Ceylan et dans l'archipel de Malaisie. Les carnets de voyage et les dessins de ces expéditions sont un hommage au monde des plantes tropicales. C'est par cette dimension esthétique que la botanique resta vivante dans les travaux de Haeckel, et c'est ainsi que se conclut une passion qui l'avait tenu sous son charme depuis l'enfance.

\section{NOTES}

1. Pour un aperçu de la vie et de l'œuvre de Haeckel voir Mario A. Di Gregorio, From Here to Eternity. Ernst Haeckel and Scientific Faith, Göttingen, Vandenhoeck \& Ruprecht, 2005 et Robert J. Richards, The Tragic Sense of Life. Ernst Haeckel and the Struggle over Evolutionary Thought, Chicago \& Londres, University of Chicago Press, 2008.

2. Ernst Haeckel, registre manuscrit, Ernst Haeckel. Stud. med. in Würzburg und Berlin Collegia Academica Verzeichnis der Vortrags-Hefte von fünf Jahren, gehört 1852-1856, Archives Ernst Haeckel à Iéna (désormais AEHI), cote B 335, fol. 17 : « Johannes Müller, né le 28.4.1858, mort le 14.7.1901 [ sic]. Le plus grand biologiste allemand du xix ${ }^{e}$ siècle fut celui qui, de tous mes professeurs, eut la plus grande influence sur moi. Lors du semestre d'été 1854 à Berlin (au tournant de mes vingt ans), il détourna ma passion pour la botanique (mon premier amour) pour l'orienter vers la zoologie (ma deuxième passion). »

3. Heinrich Schmidt (1874-1935), philosophe et exécuteur testamentaire de Ernst Haeckel. Uwe Hoßfeld, « Haeckels "Eckermann” : Heinrich Schmidt (1874-1935) », Matthias Steinbach et Stefan Gerber éd., Klassische Universität und akademische Provinz : Die Universität Jena von der Mitte des 19. bis in die 30er Jahre des 20. Jahrhunderts, Iéna, Bussert et Stadeler, 2005, p. 270-288.

4. Georg Hecht (1899-1982), biologiste et professeur au Lycée de Mersebourg. Une partie des travaux de Hecht portant sur les études botaniques de Haeckel se trouve aujourd'hui aux archives Ernst Haeckel à Iéna.

5. Ernst Haeckel, manuscrit, Lebenswege von Ernst Haeckel, AEHI, cote B 312. À propos de la botanique, voir les folios 21 à 24 . Haeckel surtitra ce chapitre: „Botanische Kinder-Studien“ (" études botaniques infantiles »), fol. 21.

6. Voir Heinrich Schmidt, Ernst Haeckel. Leben und Werke, Berlin, Deutsche Buch-Gemeinschaft, 1926, p. 53-66. Gerhard Hecht, Botanische Tätigkeit Ernst Haeckels in der Teplitzer Gegend 1852. Ein Beitrag zur Biographie eines fortschrittlichen Wissenschaftlers, Musée Oblastní, Teplice, 1974.

7. Voir Ernst Haeckel, Ausgewählte Briefwechsel. Historisch-kritische Ausgabe. Band 1 Familienkorrespondenz Februar 1839 - April 1854, édité et publié par Roman Göbel, Gerhard Müller et Claudia Taszus en collaboration avec Thomas Bach, Jens Pahnke et Kathrin Polenz, éditions Franz Steiner Verlag, Stuttgart, 2017, dans le cadre du projet d'édition de la correspondance de Haeckel : http://www.leopoldina.org/de/ueber-uns/akademien-und-forschungsvorhaben/ernsthaeckel-1834-1919-briefedition/.

8. Heinrich Schmidt éd., [Ernst Haeckel. Esquisse autobiographique], Ernst Haeckel. Gemeinverständliche Werke. Band 1, Leipzig/Berlin, Alfred Kröner Verlag/Carl Henschel Verlag, 1924, p. XXIX. Haeckel offrit en juin 1912 son „Großes Herbarium“ à l'Herbarium-Haussknecht 
(aujourd'hui à Iéna), où chaque spécimen fut classé à sa place dans ce système. Les Memorialherbarien ont été versés aux Archives Ernst Haeckel à Iéna.

9. Père : Carl Gottlob Haeckel (1771-1871), juriste et conseiller du gouvernement à Mersebourg ; Mère: Charlotte Auguste Henriette Haeckel, née Sethe (1799-1889); Frère: Karl Heinrich Christoph Benjamin Haeckel (1824-1897), juriste et juge.

10. Karl Heinrich Friedrich Gude (1814-1898), professeur et écrivain, professeur particulier de Haeckel. À propos de l'enfance de Haeckel, voir Kurt Wedekind, Die Frühprägung Ernst Haeckels, Wissenschaftliche Zeitschrift der Friedrich-Schiller-Universität Jena, $25^{\mathrm{e}}$ année, fasc. 2, Iéna, 1976, section « Mathématique et sciences naturelles », p. 133-148.

11. Ernst Haeckel, manuscrit, Tagebuch von Ernst Haeckel 1855-1858, AEHI, cote B 406, p. 8.

12. Ernst Haeckel, Memorialherbarium, Ernesti Haeckelii Herbarium primum collectum anno 1840-1843 et 1846-1850, archives Ernst Haeckel à Iéna, Cote $\mathrm{E} 1$.

13. Lebenswege von Ernst Haeckel, fol. 21. Haeckel commença en premier lieu par :

Ernst Kappe, Der kleine Botaniker oder: Anleitung, die vornehmsten Gattungen und Arten der nord- und mitteldeutschen Flora nach eigener Anschauung selbst zu bestimmen, Meurs, Librairie scolaire rhénane, $2^{\mathrm{e}}$ édition, 1843, (Haeckel-Jugendbibliothek, $\mathrm{n}^{\circ} 37=66$ ).

Martin Balduin Kittel, Taschenbuch der Flora Deutschlands: zum Gebrauche auf botanischen Excursionen , Nurernberg, Schrag, 1844.

Peter Friedrich Cürie, Anleitung die im mittleren und nördlichen Deutschland wildwachsenden Pflanzen auf eine leichte und sichere Weise durch eigene Untersuchung zu bestimmen, Kittlitz in der Oberlausitz, Zobel, $5^{\circ}$ édition, 1843, (Haeckel-Jugendbibliothek, $n^{\circ} 36=65$ ).

Wilhelm Koch, Taschenbuch der Deutschen und Schweizer Flora, Leipzig, Gebhardt et Reisland, 1848, (Haeckel-Jugendbibliothek, $\mathrm{n}^{\circ} 40=69$ ).

14. Ernst Haeckel, manuscrit, Ernst Haeckel: Tagebuch, April 1851 - Oktober 1852, AEHI, cote B 309, extrait du 5 avril 1849: «Dès ma prime jeunesse je nourris une envie et une curiosité pour les sciences naturelles et les voyages. Pour ceux-ci, cela me vient du fait que mes parents m'ont toujours emmené avec eux dans leurs voyages. Durant les sept premières années de ma vie je suis allé six fois à Hirschberg en Silésie, où mon père avait un ou plusieurs biens qu'il tenait de son père. En 1842, je partis à Bonn où je restai tout un trimestre ; en 1845 toujours de là-bas, j'allai à Heidelberg et Francfort et revins en passant par Cologne et Münster. En 1845, je passai encore, avant le grand voyage le long du Rhin, les journées de canicule à Hasserode dans le Harz chez les parents de mon premier professeur Gude, et en 1847 j'entrepris un voyage avec mon professeur Gandtner et trois de mes camarades de classe dans le Bas-Harz jusqu'au Brocken en passant par Falkenstein, Selkethal, Victorshöhe, Bodethal, Regenstein, Rübeland (où je récoltai partout des plantes) et Rothe hutte. »

15. Oscar Naumann (1814-1885), comptable, peintre et professeur de dessin au Domgymnasium de Mersebourg.

16. Johann Otto Gandtner (1822-1895), professeur au Domgymnasium de Mersebourg jusqu'à 1850.

17. Christian Ernst Weiß (1833-1890), minéralogiste, géologue et phyto-paléontologue ; Otto Victor Weber (1832-1861), étudia les mathématiques et les sciences naturelles à Halle, il y enseigna ensuite comme professeur, et travailla enfin comme inspecteur des écoles à Torgau à partir de 1857 ; Friedrich August Wilhelm Hetzer (1834-1881), étudia les mathématiques et les sciences naturelles à Halle, et occupa plus tard un poste de professeur supérieur à Hagen.

18. Victor Weber, lettre à Ernst Haeckel du 6 juillet 1852 à Halle, AEHI, cote A 16209.

19. Ernst Haeckel, Memorial-Herbarium, Centuria plantarum Thuringiacarum anno 1851 p.C.n. collecta et secundum systema Linnaei sexuale distributa ab Ernesto Haeckel, AEHI, cote E 2.

20. Christian August Friedrich Garcke (1819-1904), botaniste et éminent connaisseur des plantes cormophytes d'Allemagne. Voir August Garcke, Flora von Halle, Zweiter Teil Kryptogamen nebst einem Nachtrage zu den Phanerogamen, Berlin, Karl Wiegandt, 1856 : "On fit ainsi la découverte dans les alentours de Mersebourg, notamment grâce aux indications du Dr. Haeckel, de deux sites salins 
qui, dans la flore de Halle, représentent un très grand nombre de plantes salines situées à proximité directe de cette ville, ce qui est d'autant plus réjouissant que les sites aux alentours de Mersebourg sont les plus pauvres de la région en termes de plantes sauvages. ", p. 181.

21. Ludwig Finsterbusch (1831-1916), ami d'enfance de Haeckel.

22. Wilhelm Breitenbach, Ernst Haeckel. Ein Bild seines Lebens und seiner Arbeit, édition du Dr W. Breitenbach, Odenkirchen, 1904, p. 11.

23. Voir Lebenswege von Ernst Haeckel, fol. 22-23 : «J'avais douze ans lorsque je devins attentif aux vastes variétés de roses, de chardons, de saules et de mûres. Au début, je me mettais en colère contre ces nombreuses variations et les "mauvaises manières" de ces espèces, comme Goethe lorsqu'il qualifiait le "sexe méprisable ou négligent". Alors m'est venu le doute critique quant à savoir si les "bonnes manières" dans la nature font en sorte que les espèces soient classées très précisément, comme elles le sont si bien, les unes à côté des autres, dans les livres de systématique. En conséquence, je confectionnai à côté du Haupt-Herbarium (herbier principal), qui présentait les "bonnes espèces" en exemplaires types, joliment ordonnées et étiquetées, un petit Neben-Herbarium (herbier annexe) secret qui ne formait qu'une longue succession de toutes les variations d'inter-espèces, les "mauvaises espèces". J'ai mentionné dans la préface de la Generelle Morphologie (p. XVI) ces doutes infantiles qui avaient déjà fait leur apparition en 1846, et qui jouèrent un rôle considérable dans mon travail tout au long de ma vie. »

24. Ibid., fol. 21.

25. Lebenswege von Ernst Haeckel, fol. 21.

26. Matthias Jakob Schleiden, Die Pflanze und ihr Leben. Populäre Vorträge, Leipzig, Engelmann, 1848, (Haeckel-Jugendbibliothek, $\mathrm{n}^{\circ} 30=56$ ).

27. Ernst Haeckel, manuscrit, Föhre, Tanne, Knieholz, 6 feuillets, AEHI, cote B 387 a et Der ästhetische Einflu $\beta$ nordischer Pflanzenformationen auf den Character der Landschaft, 12 feuillets, AEHI, cote B 387 b. Le mot allemand Knieholz, littéralement bois-genou, désigne les déformations naturelles et spectaculaires de certains arbres.

28. Ernst Haeckel: Tagebuch, April 1851 - Oktober 1852, extrait daté du 17 janvier 1852.

29. Alexander von Humboldt, Ansichten der Natur, mit wissenschaftlichen Erläuterungen, $3^{\mathrm{e}}$ édition, Stuttgart et Tübingen, 1849, (Haeckel-Jugendbibliothek, $n^{\circ} 2=5$ et 6 ).

30. Voir Erika Krauße, „Haeckel: Promorphologie und ,evolutionistische ästhetische Theorie Konzept und Wirkung“, Eve-Marie Engels éd., Die Rezeption von Evolutionstheorien im 19. Jahrhundert , Francfort-sur-le-Main, Suhrkamp, 1995, p. 347-394.

31. Der ästhetische Einfluß nordischer Pflanzenformationen auf den Character der Landschaft, op. cit., p. 2 .

32. Ernst Haeckel, Lettres d'un voyageur dans l'Inde [1883 pour l'original] (trad. Dr. Ch. Letourneau), Paris, C. Reinwald, 1883.

33. Voir le chapitre des fondements méthodologiques dans Matthias Jakob Schleiden, Die Botanik als inductive Wissenschaft, $3^{\mathrm{e}}$ édition, Leipzig Wilhelm Engelmann, 1849, p. 1-162, (HaeckelJugendbibliothek, $\mathrm{n}^{\circ} 29=55$ ). À propos de la signification historique de la « botanique inductive » de Schleiden voir Karl Mägdefrau, Geschichte der Botanik. Leben und Leistung großer Forscher, Stuttgart, G. Fischer, 1973, p. 201-205.

34. Wilhelm Koch, Synopsis der Deutschen und Schweizer Flora, Leipzig, Gebhardt und Reisland, $2^{\mathrm{e}}$ édition, 1846, (Haeckel-Jugendbibliothek, $n^{\circ} 41=70-71$ ). L'exemplaire de Haeckel du premier tome porte les deux devises écrites à la main sur le plat de couverture. La citation de Schleiden est tirée de Die Pflanze und ihr Leben, op.cit., p. 1; la citation de Chamisso est extraite du poème „Beringstraße" : voir Adalbert von Chamisso, Leben und Briefe. Herausgegeben durch Julius Eduard Hitzig, Leipzig, Weidman, 1839, p. 44.

35. Wilhelm Ludwig Petermann, Das Pflanzenreich in vollständigen Beschreibungen aller wichtigen Gewächse dargestellt, nach dem natürlichen Systeme geordnet und durch naturgetreue Abbildungen erläutert, Leipzig, Eisenach, 1847, (Haeckel-Jugendbibliothek, $n^{\circ} 33=59,60$ ). 
36. Ernst Haeckel, manuscrit, Ernst Haeckel: Tagebuch, April 1851 - Oktober 1852, AEHI, cote B 309, extrait daté du 14 mars 1852. Haeckel cite l'incipit célèbre de la seconde épode d'Horace : «Beatus ille qui procul negotiis » soit « Heureux celui-là qui, loin des affaires... »

37. Ibid., extrait daté du 30 mai 1852.

38. Emploi du temps du semestre d'été : voir ibid., extrait daté du 26 avril 1852. Ernst Haeckel, manuscrit, Botanik. Vorträge von Alexander Braun (Berlin) Ernst Haeckel. Sommer 1852, AEHI, cote B 281a.

39. Lettre de Victor Weber à Ernst Haeckel, Halle, 6 juillet 1852, AEHI, cote A 16209, p. 1.

40. Ernst Haeckel : Tagebuch, April 1851 - Oktober 1852, extrait daté du 28 avril 1852.

41. Ibid., extrait daté du 26 avril 1852.

42. Voir Hecht, op. cit., p. 70-95. Ernst Haeckel, manuscrit, Ernst Haeckel. Badereise nach Teplitz im Herbst 1852 10. August - 22. September, AEHI, cote B 309 a.

43. Ernst Haeckel : Tagebuch, April 1851 - Oktober 1852, fol. $36 \mathrm{v}^{\circ}$.

44. Rudolf Albert Kölliker (1817-1905), anatomiste et physiologiste, à Wurtzbourg à partir de 1847. À propos du rapport de Haeckel à Kölliker, voir Georg Uschmann, Über die Beziehungen zwischen Albert Kölliker und Ernst Haeckel, Wissenschaftliche Zeitschrift der Friedrich-SchillerUniversität Jena, $25^{\mathrm{e}}$ édition, brochure 2, Iéna, 1976, section mathématiques et sciences naturelles, p. 125-132.

45. Ernst Haeckel, lettre à Charlotte et Carl Gottlob Haeckel, Wurtzbourg, 31 octobre - $1^{\text {er }}$ novembre 1852, AEHI, cote A 37445, p. 4.

46. Ernst Haeckel, lettre à Charlotte et Carl Gottlob Haeckel, Wurtzbourg, 6-7 novembre 1852, AEHI, cote A 37444, p. 5.

47. Joseph August Schenk (1815-1891), botaniste et paléontologue, professeur de botanique à Wurtzbourg de 1845 à 1868.

48. Ernst Haeckel, lettre à Charlotte Haeckel, Wurtzbourg, 19 novembre 1852, AEHI, cote A 37448, p. 2-3.

49. Ernst Haeckel, lettre à Charlotte et Carl Gottlob Haeckel, Wurtzbourg, 1er janvier 1853, AEHI, cote A 39332, p. 4.

50. Ernst Haeckel, lettre à Charlotte et Carl Gottlob Haeckel, Wurtzbourg, 10 mars 1853, AEHI, cote A 37461, p. 3.

51. Diederich Franz Leonhard von Schlechtendal (1794-1866), professeur de botanique à Halle à partir de 1833.

52. Ernst Haeckel, lettre à Charlotte et Carl Gottlob Haeckel, Wurtzbourg, 25-26 avril 1853, AEHI, cote A 37464, p. 1.

53. Ernst Haeckel, lettre à Charlotte et Carl Gottlob Haeckel, Wurtzbourg, 25-26 avril 1853, AEHI, cote A 37464, p. 2. La fin, en latin, signifie : « ... professeur de botanique. Ô science délicieuse! Quand m'unirai-je enfin à toi pour toujours?»

54. Ernst Haeckel, lettre à Charlotte et Carl Gottlob Haeckel, Wurtzbourg, 25-26 avril 1853, AEHI, cote A 37464, p. 4.

55. Ernst Haeckel, lettre à Charlotte et Carl Gottlob Haeckel, Wurtzbourg, 4 mai 1853, AEHI, cote A 37465, p. 4.

56. Ernst Haeckel, lettre à Charlotte et Carl Gottlob Haeckel, Wurtzbourg, $1^{\text {er }}$ juin 1853 , AEHI, cote A 41882 , p. 3.

57. Ibid., p. 2.

58. Ernst Haeckel, lettre à Charlotte et Carl Gottlob Haeckel, Wurtzbourg, 4 mai 1853, AEHI, cote A 37465, p. 6.

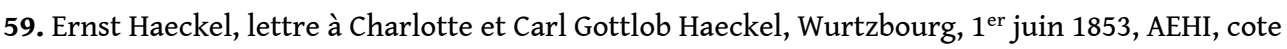
A 41882 , p. 1.

60. Ernst Haeckel, lettre à Charlotte et Carl Gottlob Haeckel, Wurtzbourg, 18 juin 1853, AEHI, cote A 37468 , p. 3. 
61. Ernst Haeckel, lettre à Charlotte et Carl Gottlob Haeckel, Wurtzbourg, 8 juillet 1853, AEHI, cote A 37471 , p. 5.

62. Ernst Haeckel, manuscrit, Skizzen aus der Entwicklungsgeschichte der Maulwurfsgrille Gryllotalpa vulgaris von Ernst Haeckel. Juli 1853. Wuerzburg [Esquisse de l'histoire du développement de la taupe-grillon (courtilière) Gryllothalpa vulgaris], AEHI, cote B 409.

63. Ernst Haeckel, lettre à Charlotte et Carl Gottlob Haeckel, Wurtzbourg, 18 juillet 1853, AEHI, cote A 37472, p. 7-8.

64. Le microscope fabriqué par Schieck à Berlin se trouve aux Archives Ernst Haeckel de Iéna. Il arbore la gravure suivante : ,Schieck in Berlin No. 668“.

65. Ernst Haeckel, lettre à Charlotte et Carl Gottlob Haeckel, Ziegenrück, 13 octobre 1853, AEHI, cote A 37478, p. 3.

66. Ernst Haeckel, lettre à Carl Gottlob Haeckel, Wurtzbourg, 21 décembre 1853, AEHI, cote A 37485 , p. 5.

67. Ibid., p. 6.

68. Ibid., p. 8.

69. Carl Christian Heinrich Haeckel (1853-1918).

70. Ernst Haeckel, lettre à Charlotte et Carl Gottlob Haeckel, Wurtzbourg, 26 janvier 1854, AEHI, cote A 37488, p. 2.

71. Ernst Haeckel, lettre à Charlotte et Carl Gottlob Haeckel, Wurtzbourg, 7 février 1854, AEHI, cote A 37489, p. 6. Wilhelm Hofmeister, Vergleichende Untersuchungen der Keimung, Entfaltung und Fruchtbildung höherer Kryptogamen (Moose, Farrn, Equisetaceen, Rhizocarpeen und Lycopodiaceen) und der Samenbildung der Coniferen, Leipzig, Friedrich Hofmeister, 1851.

72. Ernst Haeckel, lettre à Charlotte et Carl Gottlob Haeckel, Wurtzbourg, 17-18 février 1854, AEHI, cote A 37491, p. 7.

73. Tagebuch von Ernst Haeckel 1855-1858, op. cit., p. 18.

74. Johannes Peter Müller (1801-1858), physiologiste, spécialiste d'anatomie et de biologie marine comparées. Durant le semestre d'été 1854, Haeckel assista au cours de Müller sur l'anatomie et la physiologie comparées des Hommes. Voir Ernst Haeckel, manuscrit, Berlin. Sommer 1854. Johannes Müller. I. Vergleichende Anatomie. II. Physiologie des Menschen, AEHI, cote B 290.

75. Ernst Haeckel, Tagebuch von Ernst Haeckel 1855-1858, op. cit., p. 18.

76. Gottfried Koller, Das Leben des Biologen Johannes Müller 1801-1858, Stuttgart, Wissenschaftliche Verlags-Gesellschaft, 1958, p. 226-277. Laura Otis, Müller's Lab, Oxford, Oxford University Press, 2007, p. 3-41 et 190-223.

77. Adolph Freiherr von La Valette St. George (1831-1910), zoologiste et anatomiste, camarade d'étude de Haeckel à Berlin et à Wurtzbourg.

78. Ernst Haeckel, Memorialherbarium Helgoland 1854, AEHI, cote E 7.

79. Ernst Haeckel, Index animalium marinorum autumno (17/8 - 17/9) 1854 ab Ernesto Haeckel collectorum et observatorum ad insulam Helgoland, AEHI, Zoologie de Heligoland cote B 407. Le catalogue contient 200 notices.

80. Ernst Haeckel, lettre à Charlotte et Carl Gottlob Haeckel, Heligoland, 31 août 1854, AEHI, cote A 37500, p. 1.

81. Max Müller (1821-1896), chirurgien et ophtalmologiste.

82. Ernst Haeckel, lettre à Charlotte et Carl Gottlob Haeckel, Heligoland, 31 août 1854, AEHI, cote A 37500, p. 8.

83. Tagebuch von Ernst Haeckel 1855-1858, op. cit., p. 19.

84. La chaire devait couvrir aussi bien la botanique que la zoologie. Haeckel espérait cependant ne prendre en charge qu'une partie des matières, pour se consacrer ainsi uniquement à la zoologie.

85. Alexander Braun, lettre à Karl Wiebel, Berlin, 15 octobre 1860, AEHI, cote A 9034, p. 1.

86. Josef Bornmüller, lettre à Ernst Haeckel, Weimar, 12 juin 1912, AEHI, cote A 6827. 


\section{RÉSUMÉS}

Bien qu'Ernst Haeckel soit surtout connu comme zoologiste, il fit ses débuts scientifiques en botanique. Cet aspect de sa biographie scientifique est très méconnu. À la lumière du projet «Ernst Haeckel (1843-1919) : édition de la correspondance », les débuts de Haeckel en botanique ont suscité une attention croissante. Des lettres et journaux personnels inédits, ainsi que son herbier méconnu et deux articles de jeunesse, démontrent que la botanique joua un rôle déterminant dans sa formation de 1840 à 1854 . Sous l'influence de Johannes Müller, il prit ensuite la décision de faire carrière dans la zoologie. Mais son amour des plantes ne disparut jamais complètement. On examine ici en profondeur le déroulement des études botaniques de Haeckel, on montre que les plantes tinrent une grande place dans les études naturalistes du jeune Haeckel et on pose la question des raisons de sa réorientation zoologique. Haeckel continua à collecter des plantes lors de ses divers voyages, sans même que cela fût dans un but scientifique. Sa botanique devint pour une bonne part une activité esthétique, donnant lieu à de nombreux dessins et à de vives descriptions de plantes, notamment tropicales. Ajoutés à son important herbier, ils donnent une image impressionnante des activités botaniques de Haeckel.

Though Ernst Haeckel was mostly known as a zoologist, he started off into science with botany. This aspect of his scientific biography has been largely underrated. In the light of the ongoing project “Ernst Haeckel (1834-1919): Edition of Letters", Haeckel's early botanical beginnings have attracted increased attention. Hitherto unpublished letters and diaries, including his hardly known herbarium, and two early essays, support the view that botany played a pivotal role in his formal training from 1840 to 1854 . Under the influence of Johannes Müller, he first decided to pursue his scientific career as a zoologist. But his love for plants never left him completely. The course of Haeckel's botanical studies will be explored in more detail below. It will be shown that plants formed a substantial part of the young Haeckel's occupation with nature. The probable reasons for his zoological turn will be discussed. Haeckel continued collecting plants on his various travels, though without any scientific ambitions. Botany largely turned into an aesthetic enterprise, giving rise to numerous paintings and vivid descriptions of plants, especially those from the tropics. Together with his large herbarium, they provide an impressive picture of Haeckel's botanical activities.

\section{INDEX}

Mots-clés : Haeckel (Ernst), botanique, zoologie, correspondance, herbier, excursions, esthétique des plantes

Keywords : Haeckel (Ernst), botany, zoology, correspondence, herbarium, field trips, aesthetics of plants 


\section{AUTEURS}

\section{JENS PAHNKE}

Institut für Zoologie und Evolutionsforschung mit Phyletischem Museum

Ernst-Haeckel-Haus und Biologiedidaktik Friedrich-Schiller-Universität 